\title{
Leukocyte and Monocyte Counts as Predictors for Metabolic Syndrome, Hypertension and Type 2 Diabetes in Taiwan Citizens: a 9-year Longitudinal Study
}

\section{Yen-Wei Li}

Tri-Service General Hospital

\section{Tung-Wei Kao}

Tri-Service General Hospital

Chao-Yin Kuo

Tri-Service General Hospital

\section{Pi-Kai Chang}

Tri-Service General Hospital

\section{Wei-Liang Chen}

Tri-Service General Hospital

Li-Wei Wu ( $\sim$ bigmouth0825@hotmail.com )

Tri-Service General Hospital

\section{Research Article}

Keywords: Diabetes Mellitus, Hypertension, Leukocyte Count, Metabolic Syndrome

Posted Date: September 15th, 2021

DOI: https://doi.org/10.21203/rs.3.rs-141932/v2

License: (c) (i) This work is licensed under a Creative Commons Attribution 4.0 International License.

Read Full License 


\section{Abstract}

Cardiovascular disease whose correlation with metabolic syndrome and diabetes has been well established. As an aspect of preventive medicine, we conducted a 9-year longitudinal study to identify the relationship between easily measured hematologic parameters, future metabolic syndrome (MetS), hypertension and type 2 diabetes mellitus (T2DM) according to age distribution. Divided into three groups according to age (young age: $<40$, middle age: $\geqq 40$ and $<65$ and old age: $\geqq 65$ ), 10,328 participants, receiving general medical inspection at the health management centre of Tri-Service General Hospital (TSGH) in Taiwan from 2007 to 2015, had been enrolled in the present study. Through multivariate regression analyses, the hazard ratio (HR) of total and subtype leukocyte count for MetS along with its components, hypertension and T2DM were demonstrated. All MetS components revealed significant association with total leukocyte count in multivariate regression models. Total leukocyte count was associated with future MetS, hypertension and T2DM despite of age at first, but failed in adjusted HR for the old group. Additionally, monocyte count showed significant association with all the aforementioned diseases only in middle-aged subjects but failed in the other two groups. Our results explore the promising value of total leukocyte count and monocyte count to identify the high-risk subjects, especially meddle-aged ones, having MetS, hypertension, and T2DM whether in the present or future.

\section{Introduction}

CVD has become one of the main menace to human health nowadays. The complicated relationship between CVD and clustering of hyperglycemia, hyperglycemia as well as adiposity was first discovered in 1923 (1). Described as the "syndrome X" to focus on the role of insulin resistance for the aforementioned syndrome in 1988 (2), the concept of "Metabolic syndrome" (MetS) was brought by World Health Organization in 1998. The main purpose of MetS is to put emphasis on early detection of future CVD and diabetes.

Through recent epidemiologic researches, the critical part of chronic inflammation taking in both CVD and MetS had been well understood $(3,4)$. Considered as consequence of chronic inflammatory process, hypertension and diabetes also hold strong relation with cytokines and inflammatory biomarkers derived from visceral adipose tissue. Aforementioned mechanism leads to the evolution of insulin resistance, which serves as one of the major features of $\operatorname{MetS}(5,6)$.

As an empirical biomarker for acute infection, tissue injury, as well as a variety of other inflammatory circumstances, elevated leucocyte count had been demonstrated highly involved in atherosclerosis, CVD, hypertension, T2DM as well as MetS (7-10). For its easy access, it is logical for total and subtotal leukocyte counts to become a promising predictor of the diseases mentioned above. Although numerous studies had been performed to identify the relationship between leukocyte and MetS, hypertension and T2DM, respectively, most of them are cross-sectional designed. Two cohort studies with relatively extensive scale of subjects demonstrated the significant association between leukocyte and the occurrence of MetS after multivariate adjustment $(11,12)$. Nevertheless, there has been no published 
longitudinal study centering on the parts taken by leukocyte counts in future risk of these three diseasespecific groups. Take the rising prevalence according to age of MetS, hypertension and T2DM into consideration, we brought these three diseases of civilization together in a 9-year longitudinal study to identify the promising value of leukocyte counts of predicting them by age distribution.

\section{Methods And Materials}

\section{Ethics statement}

The present study was authorized by the Institutional Review Board (IRB) of Tri-Service General Hospital, National Defense Medical Center in Taiwan (\#2_106_05_144) based on the revised Helsinki Declaration. The data were collected, analyzed and used only for research purposes. Since the study used deidentified past health check records, the IRB granted a waiver of informed consent for the study. The entire methods were performed conforming to relevant regulations and guidelines

\section{Study Population}

For the present 9-year longitudinal research between 2007 to 2015, a total 52,666 participants receiving routine medical inspection at the health management centre of Tri-Service General Hospital (TSGH) in Taiwan had been randomly selected for investigation.

Information on past medical history, family history, cigarette smoking, alcoholic consumption as well as lifestyle were acquired through interviews. To eliminate the influence from possible confounding factors, we've determined exclusion criteria as the followings: subjects with past history of CVD, hypertension, T2DM $(n=2,604)$; participants with any diseases or taking any medication which may influence metabolism or hematological parameters $(n=543)$. In addition, subjects lacking data of MetS components, hematological parameters, the audiometric measurements, and the results of laboratory as well as clinical examinations $(n=37,900)$ had been excluded.

As shown in Fig. 1, we've separated the present study into two parts. First, we've organized a crosssectional observation to identify the associations between total/subtype leukocyte counts and MetS components. Second, the longitudinal part of this study had been arranged with 1,291 subjects excluded for having any history of MetS, hypertension and T2DM at baseline. Consequently, only 10,328 participants were enrolled in this part of the study. For its well documented changes for prevalence of MetS, hypertension and T2DM in different age groups, we further classified our subjects according to age distribution as young $(<40)(n=3,476)$, middle-aged ( $\geqq 40$ and $<65)(n=3,201)$, and old $(\geqq 65)(n=$ $3,651)$. The major goal of this part was to emphasize the capability for total/subtype leukocyte counts to serve as promising predictors for future risk of developing the three aforementioned diseases.

\section{Anthropometric measurements and general data}

Through a questionnaire, our trained nursing staff collected medical history, family history as well as current medications from each of our participants, respectively. While the complete physical 
examinations were performed by physicians. Waist circumference (WC) was measured in the standing position at the point halfway between the lower border of ribs and the iliac crest in a horizontal plane using a constant tension tape(13). Body mass index (BMI) was computed as weight in kilograms divided by height in meters squared $\left(\mathrm{kg} / \mathrm{m}^{2}\right)$. In sitting position through standard mercury sphygmomanometers, both diastolic blood pressure (DBP) and systolic blood pressure (SBP) were evaluated after a 5-min rest period.

\section{Laboratory evaluation}

After an overnight fast about 8 to 10 hours, all blood samples were collected through a catheter that was indwelling in a forearm vein to acquire serum levels of low and high-density lipoprotein cholesterol (LDLC; HDL-C), triglyceride (TG), total cholesterol (TC), as well as fasting plasma sugar. Blood specimens were assembled in EDTA containing tubes for every participant at the identical space. Fasting blood sugar levels were determined in duplicate by a glucose oxidase method (YSI 203 glucose analyzer, Scientific Division, Yellow Springs Instruments, Yellow Springs, $\mathrm{OH})$. Through an enzymatic method $(14,15)$, concentration of serum LDL-C and HDL-C had been determined. In addition, through dry, multilayer analytical slide method in the Fuji Dri-Chem 3000 analyzer (Fuji Photo Film, Minato-Ku, Tokyo, Japan), we've obtained concentrations of TG and TC. The total WBC count and differential count were assessed by an autoanalyzer (ADVIA 129, Bayer, Germany).

\section{Statistical analysis}

All data analysis was performed by IBM SPSS Statistics (SPSS Released 2009. PASW Statistics for Windows, Version 18.0. SPSS). Descriptive statistics were used to analyze the studied parameters and characterize the study subjects. Data were presented as numbers and percentages for qualitative data while the mean $\pm S D$ for quantitative parameters. Correlation analysis was carried out by Spearman test and Student's t test for between-group comparisons. The HRs of total/subtype leukocyte counts for each of MetS components were accomplished by multivariate linear regression models, which further investigated aforementioned relationships into the detail. For the second longitudinal part, the HRs for the three different groups according to age during the follow-up period had been performed by univariate and multivariate Cox regression models. Statistical significance was suggested by two-tailed $p$ values lower than 0.05 .

\section{Definition of metabolic syndrome}

Metabolic syndrome was diagnosed on the basis of International Diabetes Federation Global Consensus Definition(16) as central obesity (based on gender- and race-specific WC cutoffs; $\geqq 80 \mathrm{~cm}$ in women and $\geqq 90 \mathrm{~cm}$ in men for South Asians) coupled with whichever two of the subsequent four criteria as shown in Supplementary Table 1.

\section{Results}




\section{Characteristics and hematological parameters of the participants with or without metabolic syndrome}

Table 1 demonstrated the clinical characteristics of study participants classified through MetS. The participants with MetS were elder, had higher BMI, WC, SBP, DBP, body fat percentage, fasting glucose, TG, TC, LDL-C, BUN, CREA, UA as well as lower HDL-C. Significant elevation of some hematological data, including platelet count, total leukocyte count, lymphocyte count as well as neutrophil count were also identified for participants with MetS. 
Table 1

Demographic data of participants with or without metabolic syndrome.

Metabolic syndrome

Control

$P$ value

Continuous Variables

Patient profile, mean (SD)

Age (years), mean (SD)

$50.94(15.30)$

$43.84(15.77)<0.001^{\star \star}$

BMI $\left(\mathrm{kg} / \mathrm{m}^{2}\right)$, mean (SD)

$27.78(4.39)$

$22.99(3.36)$

$<0.001^{\star \star}$

WC $(\mathrm{cm})$, mean (SD)

$90.67(9.32)$

$77.36(9.93)$

$<0.001^{\star \star}$

SBP (mmHg), mean (SD)

127.27 (18.49)

$112.63(20.92)<0.001^{\star \star}$

DBP (mmHg), mean (SD)

80.94 (12.16)

$71.37(10.97)$

$<0.001$ **

Body fat percentage (\%), mean (SD)

$30.88(6.28)$

$27.07(6.86)$

$<0.001^{\star *}$

\section{Serological data, mean (SD)}

FPG (mg/dl), mean (SD)

$114.16(37.93)$

$91.57(16.13)$

$<0.001^{\star \star}$

HDL-C (mg/dl), mean (SD)

44.44 (10.73)

59.43 (15.54)

$<0.001^{\star \star}$

$\mathrm{TG}(\mathrm{mg} / \mathrm{dl})$, mean (SD)

$184.23(136.25)$

$97.41(70.35)$

$<0.001^{\star *}$

$\mathrm{TC}(\mathrm{mg} / \mathrm{dl})$, mean (SD)

193.14 (34.78)

$184.31(33.88)<0.001^{* *}$

LDL-C (mg/dl), mean (SD)

124.41 (32.23)

115.01 (30.92)

$<0.001^{\star \star}$

BUN(mg/dl), mean (SD)

$14.22(4.25)$

$13.19(4.01)$

$<0.001^{\star *}$

CREA(mg/dl), mean (SD)

$0.88(0.21)$

$0.82(0.26)$

$<0.001^{\text {** }}$

$\mathrm{UA}(\mathrm{mg} / \mathrm{dl})$, mean $(\mathrm{SD})$

6.46 (3.12)

$5.40(1.41)$

$<0.001^{\star *}$

Total Ca $(\mathrm{mg} / \mathrm{dl})$, mean (SD)

$9.20(0.40)$

$9.20(0.39)$

0.681

$\mathrm{P}(\mathrm{mg} / \mathrm{dl})$, mean (SD)

3.97 (1.19)

3.90 (1.73)

0.382

$\mathrm{r}-\mathrm{GT}(\mathrm{U} / \mathrm{L})$, mean (SD)

45.19 (63.38)

$26.59(28.76)$

$<0.001^{\star *}$

${ }^{*} \mathrm{p}<0.05,{ }^{*} \mathrm{p}<0.01$

Abbreviation:

BMI, body mass index; WC, waist circumference; SBP, systolic blood pressure; DBP, diastolic blood pressure; FPG, fasting plasma glucose; HDL-C, high-density lipoprotein cholesterol; TG, triglyceride; TC, total cholesterol; LDL-C, low-density lipoprotein cholesterol; BUN, blood urea nitrogen; CREA, creatinine; UA, uric acid; r-GT, r-glutamyl transferase; CRP, C-Reactive Protein; CA-199, carbohydrate antigen 19 - 9; AFP, alpha-fetoprotein; CEA, carcinoembryonic antigen; PSA, prostate-specific antigen; TSH, thyroid-stimulating hormone; FT4, free T4; WBC, white blood cell count; NEUT, neutrophil count; LYMP, lymphocyte count; MONO, monocyte count; EOSIN, eosinophil count; BAS, basophil count; PLT, platelet count; CV history, family history of cardiovascular disease 


\begin{tabular}{|c|c|c|c|}
\hline & Metabolic syndrome & Control & $P$ value \\
\hline Alb (g/dl), mean (SD) & $4.62(0.30)$ & $4.64(0.32)$ & 0.253 \\
\hline A/G Ratio, mean (SD) & $1.56(0.27)$ & $1.61(0.25)$ & $<0.001^{\star \star}$ \\
\hline Direct bilirubin (mg/dl), mean (SD) & $0.17(0.08)$ & $0.18(0.09)$ & 0.199 \\
\hline CRP(mg/dl), mean (SD) & $0.27(0.29)$ & $0.22(0.57)$ & 0.230 \\
\hline CA-199 (U/ml), mean (SD) & $12.21(9.62)$ & $12.40(11.55)$ & 0.798 \\
\hline AFP (ng/ml), mean (SD) & $4.32(2.07)$ & $5.06(14.10)$ & 0.215 \\
\hline CEA (ng/ml), mean (SD) & $1.78(1.13)$ & $1.57(0.92)$ & $<0.001^{\star *}$ \\
\hline PSA (ng/ml), mean (SD) & $1.26(1.73)$ & $1.19(1.34)$ & 0.468 \\
\hline TSH (uU/ml), mean (SD) & $2.41(2.70)$ & $2.20(1.90)$ & 0.056 \\
\hline FT4 (ng/dl), mean (SD) & $1.29(0.28)$ & $1.45(0.44)$ & 0.157 \\
\hline \multicolumn{4}{|l|}{ Hematological data, mean (SD) } \\
\hline WBC $\left(10^{9} / \mathrm{L}\right)$, mean $(\mathrm{SD})$ & $6.79(1.89)$ & $6.11(1.59)$ & $<0.001^{\star *}$ \\
\hline NEUT (\%), mean (SD) & $57.79(7.57)$ & $56.93(8.57)$ & $0.023^{\star}$ \\
\hline LYMP (\%), mean (SD) & $32.62(6.99)$ & $33.38(7.80)$ & $0.026^{*}$ \\
\hline MONO (\%), mean (SD) & $6.39(1.67)$ & $6.51(1.80)$ & 0.135 \\
\hline EOSIN (\%), mean (SD) & $2.66(1.85)$ & $2.62(2.09)$ & 0.623 \\
\hline BAS (\%), mean (SD) & $0.54(0.27)$ & $0.56(0.33)$ & 0.156 \\
\hline $\operatorname{PLT}\left(10^{9} / \mathrm{L}\right)$, mean $(\mathrm{SD})$ & $248.22(61.57)$ & 244.25 (57.23) & $0.048^{\star}$ \\
\hline \multicolumn{4}{|l|}{ Categorical Variables } \\
\hline Male, N (\%) & 985 (64.6) & $4251(48.3)$ & $<0.001^{\star *}$ \\
\hline CV history, N (\%) & $320(28.7)$ & $1533(24.8)$ & $0.005^{\star \star}$ \\
\hline \multicolumn{4}{|l|}{$* p<0.05, * * p<0.01$} \\
\hline \multicolumn{4}{|l|}{ Abbreviation: } \\
\hline \multicolumn{4}{|c|}{$\begin{array}{l}\text { BMI, body mass index; WC, waist circumference; SBP, systolic blood pressure; DBP, diastolic blood } \\
\text { pressure; FPG, fasting plasma glucose; HDL-C, high-density lipoprotein cholesterol; TG, triglyceride; } \\
\text { TC, total cholesterol; LDL-C, low-density lipoprotein cholesterol; BUN, blood urea nitrogen; CREA, } \\
\text { creatinine; UA, uric acid; r-GT, r-glutamyl transferase; CRP, C-Reactive Protein; CA-199, carbohydrate } \\
\text { antigen 19-9; AFP, alpha-fetoprotein; CEA, carcinoembryonic antigen; PSA, prostate-specific antigen; } \\
\text { TSH, thyroid-stimulating hormone; FT4, free T4; WBC, white blood cell count; NEUT, neutrophil count; } \\
\text { LYMP, lymphocyte count; MONO, monocyte count; EOSIN, eosinophil count; BAS, basophil count; PLT, } \\
\text { platelet count; CV history, family history of cardiovascular disease }\end{array}$} \\
\hline
\end{tabular}




\section{Association between the metabolic syndrome components and hematological parameters (white blood cell count and differential)}

We first examined relationship between MetS components and leukocyte subgroups by multivariate regression analyses. The HRs of total and subtype leukocyte counts for MetS components are shown in Table 2. Elevated total leukocyte count was significantly and positively correlated with WC, SBP, DBP, fasting glucose, and TG while negatively correlated with HDL-C. The association remained significant after twice adjustment in Model 2 (adjusted for age, sex, and BMI) and Model 3 (adjusted for age, sex, $\mathrm{BMI}$, history of CVD, TC, and CREA). Moreover, negative correlation between monocyte count and SBP, DBP, fasting glucose, TG was discovered in Model 3. 
Table 2

Multivariate hazard ratios of metabolic components by white blood cell count and differential.

\begin{tabular}{|c|c|c|c|c|c|c|}
\hline \multirow[t]{3}{*}{ Variables } & \multicolumn{6}{|l|}{ WC } \\
\hline & \multicolumn{2}{|l|}{ Model 1} & \multicolumn{2}{|l|}{ Model 2} & \multicolumn{2}{|l|}{ Model 3} \\
\hline & $\operatorname{Exp}(\beta)(95 \% \mathrm{Cl})$ & $P$ value & $\operatorname{Exp}(\beta)(95 \% \mathrm{Cl})$ & $P$ value & $\operatorname{Exp}(\beta)(95 \% \mathrm{Cl})$ & $P$ value \\
\hline WBC & $\begin{array}{l}0.180(0.930 \\
1.533)\end{array}$ & $<.001^{\star *}$ & $\begin{array}{l}0.036(0.096 \\
0.399)\end{array}$ & $0.001^{\star *}$ & $\begin{array}{l}0.036(0.094 \\
0.395)\end{array}$ & $0.002^{* *}$ \\
\hline NEUT & $\begin{array}{l}0.048(-0.012 \\
0.142)\end{array}$ & 0.097 & $\begin{array}{l}0.037(0.012 \\
0.089)\end{array}$ & $0.010^{*}$ & $\begin{array}{l}0.036(0.012 \\
0.088)\end{array}$ & $0.011^{*}$ \\
\hline LYMP & $\begin{array}{l}-0.084(-0.207 \\
-0.041)\end{array}$ & $0.003^{\star \star}$ & $\begin{array}{l}-0.038(-0.097 \\
-0.014)\end{array}$ & $0.008^{* *}$ & $\begin{array}{l}-0.037(-0.097 \\
-0.014)\end{array}$ & $0.009 * *$ \\
\hline MONO & $\begin{array}{l}0.063(0.047 \\
0.812)\end{array}$ & $0.028^{*}$ & $\begin{array}{l}-0.009(-0.255 \\
0.134)\end{array}$ & 0.542 & $\begin{array}{l}-0.008(-0.249 \\
0.139)\end{array}$ & 0.578 \\
\hline EOSIN & $\begin{array}{l}0.085(0.165 \\
0.794)\end{array}$ & $0.003^{* *}$ & $\begin{array}{l}0.000(-0.158 \\
0.159)\end{array}$ & 0.993 & $\begin{array}{l}0.002(-0.150 \\
0.167)\end{array}$ & 0.916 \\
\hline BAS & $\begin{array}{l}-0.088(-5.090 \\
-1.128)\end{array}$ & $0.002^{\star *}$ & $\begin{array}{l}-0.013(-1.453 \\
0.539)\end{array}$ & 0.368 & $\begin{array}{l}-0.013(-1.438 \\
0.552)\end{array}$ & 0.383 \\
\hline \multirow[t]{3}{*}{ Variables } & \multicolumn{6}{|l|}{ SBP } \\
\hline & \multicolumn{2}{|l|}{ Model 1} & \multicolumn{2}{|l|}{ Model 2} & \multicolumn{2}{|l|}{ Model 3} \\
\hline & $\operatorname{Exp}(\beta)(95 \% \mathrm{Cl})$ & $P$ value & $\operatorname{Exp}(\beta)(95 \% \mathrm{Cl})$ & $P$ value & $\operatorname{Exp}(\beta)(95 \% \mathrm{Cl})$ & $P$ value \\
\hline WBC & $\begin{array}{l}0.129(1.012 \\
1.783)\end{array}$ & $<.001^{\star *}$ & $\begin{array}{l}0.151(1.280 \\
2.004)\end{array}$ & $<.001^{\star \star}$ & $\begin{array}{l}0.148(1.244 \\
1.968)\end{array}$ & $\dot{0.001 * *}$ \\
\hline NEUT & $\begin{array}{l}0.015(-0.062 \\
0.126)\end{array}$ & 0.509 & $\begin{array}{l}0.034(-0.015 \\
0.162)\end{array}$ & 0.103 & $\begin{array}{l}0.038(-0.006 \\
0.172)\end{array}$ & 0.066 \\
\hline
\end{tabular}

${ }^{*} \mathrm{p}<0.05, * * \mathrm{p}<0.01$

Abbreviation:

WC, waist circumference; SBP, systolic blood pressure; DBP, diastolic blood pressure; FPG, fasting plasma glucose; TG, triglyceride; HDL-C, high-density lipoprotein cholesterol; WBC, white blood cell count; NEUT, neutrophil count; LYMP, lymphocyte count; MONO, monocyte count; EOSIN, eosinophil count; BAS, basophil count

Adjusted covariates:

Model 1 = Unadjusted.

Model 2 = Model $1+$ Age, Sex and body mass index (BMI).

Model 3 = Model 2 + Familyhistory2 (family history of cardiovascular disease), TC (total cholesterol), CREA (creatinine) 


\begin{tabular}{|c|c|c|c|c|c|c|}
\hline \multirow[t]{3}{*}{ Variables } & \multicolumn{6}{|l|}{ WC } \\
\hline & \multicolumn{2}{|l|}{ Model 1} & \multicolumn{2}{|l|}{ Model 2} & \multicolumn{2}{|l|}{ Model 3} \\
\hline & $\operatorname{Exp}(\beta)(95 \% \mathrm{Cl})$ & $P$ value & $\operatorname{Exp}(\beta)(95 \% \mathrm{Cl})$ & $P$ value & $\operatorname{Exp}(\beta)(95 \% \mathrm{Cl})$ & $P$ value \\
\hline LYMP & $\begin{array}{l}-0.012(-0.130 \\
0.075)\end{array}$ & 0.598 & $\begin{array}{l}-0.005(-0.108 \\
0.085)\end{array}$ & 0.812 & $\begin{array}{l}-0.008(-0.114 \\
0.078)\end{array}$ & 0.714 \\
\hline MONO & $\begin{array}{l}-0.006(-0.493 \\
0.379)\end{array}$ & 0.798 & $\begin{array}{l}-0.067(-1.082 \\
-0.254)\end{array}$ & $0.002^{\star \star}$ & $\begin{array}{l}-0.071(-1.124 \\
-0.296)\end{array}$ & $0.001^{\star *}$ \\
\hline EOSIN & $\begin{array}{l}0.000(-0.366 \\
0.375)\end{array}$ & 0.982 & $\begin{array}{l}-0.050(-0.779 \\
-0.076)\end{array}$ & $0.017^{*}$ & $\begin{array}{l}-0.054(-0.810 \\
-0.106)\end{array}$ & $0.011^{\star}$ \\
\hline BAS & $\begin{array}{l}-0.073(-6.173, \\
-1.581)\end{array}$ & $0.001^{\star \star}$ & $\begin{array}{l}-0.073(-6.009 \\
-1.703)\end{array}$ & $<0.001$ * & $\begin{array}{l}-0.074(-6.077 \\
-1.778)\end{array}$ & $<.001^{* *}$ \\
\hline \multirow[t]{3}{*}{ Variables } & \multicolumn{6}{|l|}{ DBP } \\
\hline & \multicolumn{2}{|l|}{ Model 1} & \multicolumn{2}{|l|}{ Model 2} & \multicolumn{2}{|l|}{ Model 3} \\
\hline & $\operatorname{Exp}(\beta)(95 \% \mathrm{Cl})$ & $P$ value & $\operatorname{Exp}(\beta)(95 \% \mathrm{Cl})$ & $P$ value & $\operatorname{Exp}(\beta)(95 \% \mathrm{Cl})$ & $P$ value \\
\hline WBC & $\begin{array}{l}0.133(0.693 \\
1.196)\end{array}$ & $<.001 * \star$ & $\begin{array}{l}0.156(0.868 \\
1.346)\end{array}$ & $<.001$ * & $\begin{array}{l}0.150(0.826 \\
1.302)\end{array}$ & $\begin{array}{l}<.001^{* *} \\
\end{array}$ \\
\hline NEUT & $\begin{array}{l}0.009(-0.048 \\
0.073)\end{array}$ & 0.691 & $\begin{array}{l}0.028(-0.019 \\
0.096)\end{array}$ & 0.187 & $\begin{array}{l}0.033(-0.011 \\
0.104)\end{array}$ & 0.113 \\
\hline LYMP & $\begin{array}{l}-0.008(-0.078 \\
0.054)\end{array}$ & 0.722 & $\begin{array}{l}-0.002(-0.065 \\
0.060)\end{array}$ & 0.937 & $\begin{array}{l}-0.007(-0.072 \\
0.052)\end{array}$ & 0.756 \\
\hline MONO & $\begin{array}{l}-0.007(-0.327 \\
0.236)\end{array}$ & 0.749 & $\begin{array}{l}-0.066(-0.695 \\
-0.156)\end{array}$ & $0.002^{\star \star}$ & $\begin{array}{l}-0.069(-0.713 \\
-0.177)\end{array}$ & $0.001^{\star *}$ \\
\hline EOSIN & $\begin{array}{l}0.006(-0.204 \\
0.274)\end{array}$ & 0.774 & $\begin{array}{l}-0.042(-0.462 \\
-0.005)\end{array}$ & $0.046^{\star}$ & $\begin{array}{l}-0.044(-0.467 \\
-0.012)\end{array}$ & $0.039 *$ \\
\hline
\end{tabular}

${ }^{*} \mathrm{p}<0.05, * * \mathrm{p}<0.01$

Abbreviation:

WC, waist circumference; SBP, systolic blood pressure; DBP, diastolic blood pressure; FPG, fasting plasma glucose; TG, triglyceride; HDL-C, high-density lipoprotein cholesterol; WBC, white blood cell count; NEUT, neutrophil count; LYMP, lymphocyte count; MONO, monocyte count; EOSIN, eosinophil count; BAS, basophil count

Adjusted covariates:

Model 1 = Unadjusted.

Model 2 = Model $1+$ Age, Sex and body mass index (BMI).

Model 3 = Model 2 + Familyhistory2 (family history of cardiovascular disease), TC (total cholesterol), CREA (creatinine) 


\begin{tabular}{|c|c|c|c|c|c|c|}
\hline \multirow[t]{3}{*}{ Variables } & \multicolumn{6}{|l|}{ WC } \\
\hline & \multicolumn{2}{|l|}{ Model 1} & \multicolumn{2}{|l|}{ Model 2} & \multicolumn{2}{|l|}{ Model 3} \\
\hline & $\operatorname{Exp}(\beta)(95 \% \mathrm{Cl})$ & $P$ value & $\operatorname{Exp}(\beta)(95 \% \mathrm{Cl})$ & $P$ value & $\operatorname{Exp}(\beta)(95 \% \mathrm{Cl})$ & $P$ value \\
\hline BAS & $\begin{array}{l}-0.044(-2.997 \\
-0.028)\end{array}$ & $0.046^{\star}$ & $\begin{array}{l}-0.044(-2.904 \\
-0.101)\end{array}$ & $0.036^{\star}$ & $\begin{array}{l}-0.046(-2.957 \\
-0.173)\end{array}$ & $0.028^{*}$ \\
\hline \multirow[t]{3}{*}{ Variables } & \multicolumn{6}{|l|}{ FPG } \\
\hline & \multicolumn{2}{|l|}{ Model 1} & \multicolumn{2}{|l|}{ Model 2} & \multicolumn{2}{|l|}{ Model 3} \\
\hline & $\operatorname{Exp}(\beta)(95 \% \mathrm{Cl})$ & $P$ value & $\operatorname{Exp}(\beta)(95 \% \mathrm{Cl})$ & $P$ value & $\operatorname{Exp}(\beta)(95 \% \mathrm{Cl})$ & $P$ value \\
\hline WBC & $\begin{array}{l}0.074(0.515 \\
1.574)\end{array}$ & $<0.001 * *$ & $\begin{array}{l}0.106(0.990 \\
2.019)\end{array}$ & $<.001^{\star *}$ & $\begin{array}{l}0.102(0.930 \\
1.957)\end{array}$ & $<.001^{* *}$ \\
\hline NEUT & $\begin{array}{l}0.019(-0.070 \\
0.170)\end{array}$ & 0.415 & $\begin{array}{l}0.018(-0.066 \\
0.163)\end{array}$ & 0.409 & $\begin{array}{l}0.022(-0.057 \\
0.172)\end{array}$ & 0.326 \\
\hline LYMP & $\begin{array}{l}-0.024(-0.199 \\
0.061)\end{array}$ & 0.299 & $\begin{array}{l}-0.004(-0.135 \\
0.113)\end{array}$ & 0.858 & $\begin{array}{l}-0.007(-0.145 \\
0.103)\end{array}$ & 0.737 \\
\hline MONO & $\begin{array}{l}-0.004(-0.597 \\
0.500)\end{array}$ & 0.862 & $\begin{array}{l}-0.046(-1.077 \\
-0.021)\end{array}$ & $0.042^{\star}$ & $\begin{array}{l}-0.047(-1.095 \\
-0.040)\end{array}$ & $0.035^{\star}$ \\
\hline EOSIN & $\begin{array}{l}0.019(-0.281 \\
0.667)\end{array}$ & 0.424 & $\begin{array}{l}-0.016(-0.623 \\
0.292)\end{array}$ & 0.479 & $\begin{array}{l}-0.015(-0.617 \\
0.298)\end{array}$ & 0.494 \\
\hline BAS & $\begin{array}{l}-0.014(-3.834 \\
2.032)\end{array}$ & 0.547 & $\begin{array}{l}-0.020(-4.065 \\
1.514)\end{array}$ & 0.370 & $\begin{array}{l}-0.021(-4.162 \\
1.404)\end{array}$ & 0.331 \\
\hline \multirow[t]{3}{*}{ Variables } & \multicolumn{6}{|l|}{ TG } \\
\hline & \multicolumn{2}{|l|}{ Model 1} & \multicolumn{2}{|l|}{ Model 2} & \multicolumn{2}{|l|}{ Model 3} \\
\hline & $\operatorname{Exp}(\beta)(95 \% \mathrm{Cl})$ & $P$ value & $\operatorname{Exp}(\beta)(95 \% \mathrm{Cl})$ & $P$ value & $\operatorname{Exp}(\beta)(95 \% \mathrm{Cl})$ & $P$ value \\
\hline \multicolumn{7}{|c|}{${ }^{*} p<0.05, * \star p<0.01$} \\
\hline \multicolumn{7}{|c|}{ Abbreviation: } \\
\hline \multicolumn{7}{|c|}{$\begin{array}{l}\text { WC, waist circumference; SBP, systolic blood pressure; DBP, diastolic blood pressure; FPG, fasting } \\
\text { plasma glucose; TG, triglyceride; HDL-C, high-density lipoprotein cholesterol; WBC, white blood cell } \\
\text { count; NEUT, neutrophil count; LYMP, lymphocyte count; MONO, monocyte count; EOSIN, eosinophil } \\
\text { count; BAS, basophil count }\end{array}$} \\
\hline \multicolumn{7}{|c|}{ Adjusted covariates: } \\
\hline \multicolumn{7}{|c|}{ Model 1 = Unadjusted. } \\
\hline \multicolumn{7}{|c|}{ Model 2 = Model $1+$ Age, Sex and body mass index (BMI). } \\
\hline $\begin{array}{l}\text { Model } 3=1 \\
\text { CREA (crea }\end{array}$ & $\begin{array}{l}\text { lodel } 2 \text { + Familyhis } \\
\text { inine) }\end{array}$ & y2 (fami & history of cardio & ular dis & se), TC (total chc & terol), \\
\hline
\end{tabular}




\begin{tabular}{|c|c|c|c|c|c|c|}
\hline \multirow[t]{3}{*}{ Variables } & \multicolumn{6}{|l|}{ WC } \\
\hline & \multicolumn{2}{|l|}{ Model 1} & \multicolumn{2}{|l|}{ Model 2} & \multicolumn{2}{|l|}{ Model 3} \\
\hline & $\operatorname{Exp}(\beta)(95 \% \mathrm{Cl})$ & $P$ value & $\operatorname{Exp}(\beta)(95 \% \mathrm{Cl})$ & $P$ value & $\operatorname{Exp}(\beta)(95 \% \mathrm{Cl})$ & $P$ value \\
\hline WBC & $\begin{array}{l}0.172 \text { (7.668, } \\
11.627)\end{array}$ & $\begin{array}{l}<.001^{\star *} \\
\end{array}$ & $\begin{array}{l}0.189(8.727 \\
12.519)\end{array}$ & $<.001^{\star *}$ & $\begin{array}{l}0.176(8.036 \\
11.727)\end{array}$ & $\dot{<} 001^{\star \star}$ \\
\hline NEUT & $\begin{array}{l}-0.029(-0.804 \\
0.156)\end{array}$ & 0.186 & $\begin{array}{l}-0.012(-0.590 \\
0.324)\end{array}$ & 0.567 & $\begin{array}{l}0.003(-0.412 \\
0.482)\end{array}$ & 0.877 \\
\hline LYMP & $\begin{array}{l}0.024(-0.238 \\
0.806)\end{array}$ & 0.287 & $\begin{array}{l}0.031(-0.119 \\
0.872)\end{array}$ & 0.136 & $\begin{array}{l}0.015(-0.307 \\
0.663)\end{array}$ & 0.472 \\
\hline MONO & $\begin{array}{l}0.018(-1.307 \\
3.150)\end{array}$ & 0.418 & $\begin{array}{l}-0.040(-4.205 \\
0.082)\end{array}$ & 0.059 & $\begin{array}{l}-0.046(-4.461 \\
-0.275)\end{array}$ & $0.027^{\star}$ \\
\hline EOSIN & $\begin{array}{l}0.018(-1.097 \\
2.690)\end{array}$ & 0.409 & $\begin{array}{l}-0.029(-3.101 \\
0.531)\end{array}$ & 0.165 & $\begin{array}{l}-0.023(-2.773 \\
0.782)\end{array}$ & 0.272 \\
\hline BAS & $\begin{array}{l}-0.023(-17.937 \\
5.571)\end{array}$ & 0.302 & $\begin{array}{l}-0.023(-17.484 \\
4.790)\end{array}$ & 0.264 & $\begin{array}{l}-0.033(-19.741 \\
1.991)\end{array}$ & 0.109 \\
\hline \multirow[t]{3}{*}{ Variables } & \multicolumn{6}{|l|}{ HDL-C } \\
\hline & \multicolumn{2}{|l|}{ Model 1} & \multicolumn{2}{|l|}{ Model 2} & \multicolumn{2}{|l|}{ Model 3} \\
\hline & $\operatorname{Exp}(\beta)(95 \% \mathrm{Cl})$ & $P$ value & $\operatorname{Exp}(\beta)(95 \% \mathrm{Cl})$ & $P$ value & $\operatorname{Exp}(\beta)(95 \% \mathrm{Cl})$ & $P$ value \\
\hline WBC & $\begin{array}{l}-0.182(-2.016 \\
-1.252)\end{array}$ & $\begin{array}{l}<.001^{\star *} \\
\end{array}$ & $\begin{array}{l}-0.170(-1.880 \\
-1.173)\end{array}$ & $<.001^{\star * *}$ & $\begin{array}{l}-0.181(-1.972, \\
-1.281)\end{array}$ & $\begin{array}{l}<.001^{* *} \\
\end{array}$ \\
\hline NEUT & $\begin{array}{l}0.005(-0.076 \\
0.092)\end{array}$ & 0.849 & $\begin{array}{l}-0.032(-0.132 \\
0.020)\end{array}$ & 0.151 & $\begin{array}{l}-0.014(-0.101 \\
0.050)\end{array}$ & 0.505 \\
\hline LYMP & $\begin{array}{l}0.019(-0.055 \\
0.126)\end{array}$ & 0.444 & $\begin{array}{l}0.022(-0.039 \\
0.125)\end{array}$ & 0.308 & $\begin{array}{l}0.005(-0.071 \\
0.090)\end{array}$ & 0.819 \\
\hline \multicolumn{7}{|c|}{${ }^{*} \mathrm{p}<0.05,{ }^{* \star} \mathrm{p}<0.01$} \\
\hline \multicolumn{7}{|c|}{ Abbreviation: } \\
\hline \multicolumn{7}{|c|}{$\begin{array}{l}\text { WC, waist circumference; SBP, systolic blood pressure; DBP, diastolic blood pressure; FPG, fasting } \\
\text { plasma glucose; TG, triglyceride; HDL-C, high-density lipoprotein cholesterol; WBC, white blood cell } \\
\text { count; NEUT, neutrophil count; LYMP, lymphocyte count; MONO, monocyte count; EOSIN, eosinophil } \\
\text { count; BAS, basophil count }\end{array}$} \\
\hline \multicolumn{7}{|c|}{ Adjusted covariates: } \\
\hline \multicolumn{7}{|c|}{ Model 1 = Unadjusted. } \\
\hline \multicolumn{7}{|c|}{ Model 2 = Model $1+$ Age, Sex and body mass index (BMI). } \\
\hline $\begin{array}{l}\text { Model } 3=1 \\
\text { CREA (crea }\end{array}$ & $\begin{array}{l}\text { del } 2 \text { + Familyhi } \\
\text { ine) }\end{array}$ & 2 (fam & history of cardic & cular dis & se), TC (total ch & sterol), \\
\hline
\end{tabular}




\begin{tabular}{|c|c|c|c|c|c|c|}
\hline \multirow[t]{3}{*}{ Variables } & \multicolumn{6}{|l|}{ WC } \\
\hline & \multicolumn{2}{|l|}{ Model 1} & \multicolumn{2}{|l|}{ Model 2} & \multicolumn{2}{|l|}{ Model 3} \\
\hline & $\operatorname{Exp}(\beta)(95 \% \mathrm{Cl})$ & $P$ value & $\operatorname{Exp}(\beta)(95 \% \mathrm{Cl})$ & $P$ value & $\operatorname{Exp}(\beta)(95 \% \mathrm{Cl})$ & $P$ value \\
\hline MONO & $\begin{array}{l}-0.032(-0.650, \\
0.125)\end{array}$ & 0.184 & $\begin{array}{l}0.032(-0.092 \\
0.619)\end{array}$ & 0.147 & $\begin{array}{l}0.025(-0.148 \\
0.552)\end{array}$ & 0.257 \\
\hline EOSIN & $\begin{array}{l}-0.065(-0.773, \\
-0.122)\end{array}$ & $0.007^{\star \star}$ & $\begin{array}{l}0.007(-0.253 \\
0.346)\end{array}$ & 0.761 & $\begin{array}{l}0.011(-0.218 \\
0.371)\end{array}$ & 0.610 \\
\hline BAS & $\begin{array}{l}0.049(0.078 \\
4.106)\end{array}$ & $0.042^{\star}$ & $\begin{array}{l}0.051(0.340 \\
3.984)\end{array}$ & $0.020^{*}$ & $\begin{array}{l}0.041(-0.060 \\
3.519)\end{array}$ & 0.058 \\
\hline \multicolumn{7}{|c|}{${ }^{\star} \mathrm{p}<0.05,{ }^{\star *} \mathrm{p}<0.01$} \\
\hline \multicolumn{7}{|c|}{ Abbreviation: } \\
\hline \multicolumn{7}{|c|}{$\begin{array}{l}\text { WC, waist circumference; SBP, systolic blood pressure; DBP, diastolic blood pressure; FPG, fasting } \\
\text { plasma glucose; TG, triglyceride; HDL-C, high-density lipoprotein cholesterol; WBC, white blood cell } \\
\text { count; NEUT, neutrophil count; LYMP, lymphocyte count; MONO, monocyte count; EOSIN, eosinophil } \\
\text { count; BAS, basophil count }\end{array}$} \\
\hline \multicolumn{7}{|c|}{ Adjusted covariates: } \\
\hline \multicolumn{7}{|c|}{ Model 1 = Unadjusted. } \\
\hline \multicolumn{7}{|c|}{ Model 2 = Model $1+$ Age, Sex and body mass index (BMI). } \\
\hline \multicolumn{7}{|c|}{$\begin{array}{l}\text { Model } 3=\text { Model } 2+\text { Familyhistory } 2 \text { (family history of cardiovascular disease), TC (total cholesterol), } \\
\text { CREA (creatinine) }\end{array}$} \\
\hline
\end{tabular}

\section{Association between the risk of future metabolic syndrome, hypertension, and type 2 diabetes mellitus as well as total and subtypes counts of white blood cell}

To further investigate the role of leukocyte and its subtypes in MetS, hypertension and T2DM, multivariate Cox regression had been performed to figure out the HRs for future development of MetS, hypertension, and T2DM by age distribution $(<40,40-64, \geq 65)$.

For subjects aged < 40-year-old (Table 3), significant association between total leukocyte count and MetS, hypertension and T2DM was found for the entire three models except failing to do so in MetS group of Model 2 and T2DM group of Model 3. Significant difference for monocyte count in both MetS and hypertension groups was also noted with exception of hypertension group in Model 3. 
Table 3

Multivariate hazard ratios of future metabolic syndrome, hypertension and type 2 diabetes mellitus by white blood cell count and differential. (Age $=1-39)$

\begin{tabular}{|c|c|c|c|c|c|c|}
\hline \multirow[t]{3}{*}{ Variables } & \multicolumn{6}{|l|}{ MetS } \\
\hline & \multicolumn{2}{|l|}{ Model 1} & \multicolumn{2}{|l|}{ Model 2} & \multicolumn{2}{|l|}{ Model 3} \\
\hline & $\operatorname{Exp}(\beta)(95 \% \mathrm{Cl})$ & $P$ value & $\operatorname{Exp}(\beta)(95 \% \mathrm{Cl})$ & $P$ value & $\operatorname{Exp}(\beta)(95 \% \mathrm{Cl})$ & $P$ value \\
\hline WBC & $\begin{array}{l}1.247(1.180 \\
1.318)\end{array}$ & $<.001^{\star *}$ & $\begin{array}{l}1.068(0.992, \\
1.149)\end{array}$ & 0.082 & $\begin{array}{l}1.149(1.025 \\
1.289)\end{array}$ & $0.017^{\star}$ \\
\hline NEUT & $\begin{array}{l}1.025(1.008 \\
1.042)\end{array}$ & $0.004^{\star *}$ & $\begin{array}{l}1.015(0.995 \\
1.035)\end{array}$ & 0.135 & $\begin{array}{l}1.017(0.982, \\
1.054)\end{array}$ & 0.335 \\
\hline LYMP & $\begin{array}{l}0.975(0.957 \\
0.993)\end{array}$ & 0.006 ** & $\begin{array}{l}0.991 \text { (0.971, } \\
1.012)\end{array}$ & 0.412 & $\begin{array}{l}0.991 \text { (0.956, } \\
1.027)\end{array}$ & 0.613 \\
\hline MONO & $\begin{array}{l}0.851(0.778 \\
0.931)\end{array}$ & $<.001^{\star \star}$ & $\begin{array}{l}0.801(0.728 \\
0.882)\end{array}$ & $\begin{array}{l}< \\
0.001^{\star *}\end{array}$ & $\begin{array}{l}0.851(0.725 \\
0.999)\end{array}$ & $0.048^{\star}$ \\
\hline EOSIN & $\begin{array}{l}1.053(0.989 \\
1.120)\end{array}$ & 0.107 & $\begin{array}{l}1.061(0.979 \\
1.150)\end{array}$ & 0.149 & $\begin{array}{l}1.008(0.842 \\
1.206)\end{array}$ & 0.935 \\
\hline BAS & $\begin{array}{l}0.551(0.294 \\
1.035)\end{array}$ & 0.064 & $\begin{array}{l}0.685(0.357 \\
1.318)\end{array}$ & 0.257 & $\begin{array}{l}0.655(0.234 \\
1.832)\end{array}$ & 0.420 \\
\hline \multirow[t]{3}{*}{ Variables } & \multicolumn{6}{|l|}{ HTN } \\
\hline & \multicolumn{2}{|l|}{ Model 1} & \multicolumn{2}{|l|}{ Model 2} & \multicolumn{2}{|l|}{ Model 3} \\
\hline & $\operatorname{Exp}(\beta)(95 \% \mathrm{Cl})$ & $P$ value & $\operatorname{Exp}(\beta)(95 \% \mathrm{Cl})$ & $P$ value & $\operatorname{Exp}(\beta)(95 \% \mathrm{Cl})$ & $P$ value \\
\hline WBC & $\begin{array}{l}1.214(1.149 \\
1.282)\end{array}$ & $<_{0.001 * \star}$ & $\begin{array}{l}1.085(1.014 \\
1.162)\end{array}$ & $0.019 *$ & $\begin{array}{l}1.228(1.098, \\
1.373)\end{array}$ & $<.001^{\star *}$ \\
\hline NEUT & $\begin{array}{l}1.016(0.999 \\
1.033)\end{array}$ & 0.057 & $\begin{array}{l}1.005(0.987 \\
1.024)\end{array}$ & 0.574 & $\begin{array}{l}0.983(0.951 \\
1.015)\end{array}$ & 0.289 \\
\hline
\end{tabular}

${ }^{*} \mathrm{p}<0.05,{ }^{*} \mathrm{p}<0.01$

Abbreviation:

MetS, metabolic syndrome; HTN, hypertension; DM, diabetes mellitus; WBC, white blood cell count; NEUT, neutrophil count; LYMP, lymphocyte count; MONO, monocyte count; EOSIN, eosinophil count; BAS, basophil count

Adjusted covariates:

Model 1 = Unadjusted .

Model 2 = Model $1+$ Age, Sex and body mass index (BMI).

Model 3 = Model 2 + Familyhistory2 (family history of cardiovascular disease), TC (total cholesterol), CREA (creatinine) 


\begin{tabular}{|c|c|c|c|c|c|c|}
\hline \multirow[t]{3}{*}{ Variables } & \multicolumn{6}{|l|}{ MetS } \\
\hline & \multicolumn{2}{|l|}{ Model 1} & \multicolumn{2}{|l|}{ Model 2} & \multicolumn{2}{|l|}{ Model 3} \\
\hline & $\operatorname{Exp}(\beta)(95 \% \mathrm{Cl})$ & $P$ value & $\operatorname{Exp}(\beta)(95 \% \mathrm{Cl})$ & $P$ value & $\operatorname{Exp}(\beta)(95 \% \mathrm{Cl})$ & $P$ value \\
\hline LYMP & $\begin{array}{l}0.990(0.972 \\
1.008)\end{array}$ & 0.284 & $\begin{array}{l}1.004(0.985 \\
1.024)\end{array}$ & 0.677 & $\begin{array}{l}1.029(0.995 \\
1.064)\end{array}$ & 0.099 \\
\hline MONO & $\begin{array}{l}0.816(0.745 \\
0.894)\end{array}$ & $<.001^{\star *}$ & $\begin{array}{l}0.832(0.756 \\
0.915)\end{array}$ & $<.001^{\star \star}$ & $\begin{array}{l}0.936(0.806 \\
1.086)\end{array}$ & 0.383 \\
\hline EOSIN & $\begin{array}{l}1.001(0.930 \\
1.078)\end{array}$ & 0.970 & $\begin{array}{l}0.981(0.897 \\
1.072)\end{array}$ & 0.669 & $\begin{array}{l}0.869(0.722 \\
1.046)\end{array}$ & 0.137 \\
\hline BAS & $\begin{array}{l}0.544(0.293 \\
1.013)\end{array}$ & 0.055 & $\begin{array}{l}0.536(0.285 \\
1.008)\end{array}$ & 0.053 & $\begin{array}{l}0.888(0.355 \\
2.222)\end{array}$ & 0.799 \\
\hline \multirow[t]{3}{*}{ Variables } & \multicolumn{6}{|l|}{ Type 2 DM } \\
\hline & \multicolumn{2}{|l|}{ Model 1} & \multicolumn{2}{|l|}{ Model 2} & \multicolumn{2}{|l|}{ Model 3} \\
\hline & $\operatorname{Exp}(\beta)(95 \% \mathrm{Cl})$ & $P$ value & $\operatorname{Exp}(\beta)(95 \% \mathrm{Cl})$ & $P$ value & $\operatorname{Exp}(\beta)(95 \% \mathrm{Cl})$ & $P$ value \\
\hline WBC & $\begin{array}{l}1.277(1.134 \\
1.439)\end{array}$ & $<.001^{\star *}$ & $\begin{array}{l}1.176(1.003 \\
1.380)\end{array}$ & $0.046^{*}$ & $\begin{array}{l}1.246(0.942 \\
1.649)\end{array}$ & 0.123 \\
\hline NEUT & $\begin{array}{l}1.026(0.979 \\
1.076)\end{array}$ & 0.286 & $\begin{array}{l}1.039(0.983 \\
1.098)\end{array}$ & 0.174 & $\begin{array}{l}1.022(0.920 \\
1.135)\end{array}$ & 0.688 \\
\hline LYMP & $\begin{array}{l}0.976(0.925 \\
1.029)\end{array}$ & 0.373 & $\begin{array}{l}0.971(0.917 \\
1.029)\end{array}$ & 0.321 & $\begin{array}{l}0.973(0.874 \\
1.083)\end{array}$ & 0.615 \\
\hline MONO & $\begin{array}{l}0.874(0.678 \\
1.126)\end{array}$ & 0.296 & $\begin{array}{l}0.831(0.630 \\
1.098)\end{array}$ & 0.193 & $\begin{array}{l}1.040(0.693 \\
1.559)\end{array}$ & 0.851 \\
\hline EOSIN & $\begin{array}{l}0.967(0.767 \\
1.219)\end{array}$ & 0.774 & $\begin{array}{l}0.885(0.646 \\
1.212)\end{array}$ & 0.446 & $\begin{array}{l}1.047(0.558 \\
1.965)\end{array}$ & 0.886 \\
\hline
\end{tabular}

* $p<0.05, * * p<0.01$

Abbreviation:

MetS, metabolic syndrome; HTN, hypertension; DM, diabetes mellitus; WBC, white blood cell count; NEUT, neutrophil count; LYMP, lymphocyte count; MONO, monocyte count; EOSIN, eosinophil count; BAS, basophil count

Adjusted covariates:

Model 1 = Unadjusted.

Model 2 = Model $1+$ Age, Sex and body mass index (BMI).

Model 3 = Model 2 + Familyhistory2 (family history of cardiovascular disease), TC (total cholesterol), CREA (creatinine) 


\begin{tabular}{|c|c|c|c|c|c|c|}
\hline \multirow[t]{3}{*}{ Variables } & \multicolumn{6}{|l|}{ MetS } \\
\hline & \multicolumn{2}{|l|}{ Model 1} & \multicolumn{2}{|l|}{ Model 2} & \multicolumn{2}{|l|}{ Model 3} \\
\hline & $\operatorname{Exp}(\beta)(95 \% \mathrm{Cl})$ & $P$ value & $\operatorname{Exp}(\beta)(95 \% \mathrm{Cl})$ & $P$ value & $\operatorname{Exp}(\beta)(95 \% \mathrm{Cl})$ & $P$ value \\
\hline BAS & $\begin{array}{l}1.364(0.296 \\
6.284)\end{array}$ & 0.691 & $\begin{array}{l}1.295(0.222 \\
7.543)\end{array}$ & 0.774 & $\begin{array}{l}3.581(0.200 \\
63.981)\end{array}$ & 0.386 \\
\hline \multicolumn{7}{|c|}{${ }^{*} \mathrm{p}<0.05, * * \mathrm{p}<0.01$} \\
\hline \multicolumn{7}{|c|}{ Abbreviation: } \\
\hline \multicolumn{7}{|c|}{$\begin{array}{l}\text { MetS, metabolic syndrome; HTN, hypertension; DM, diabetes mellitus; WBC, white blood cell count; } \\
\text { NEUT, neutrophil count; LYMP, lymphocyte count; MONO, monocyte count; EOSIN, eosinophil count; } \\
\text { BAS, basophil count }\end{array}$} \\
\hline \multicolumn{7}{|c|}{ Adjusted covariates: } \\
\hline \multicolumn{7}{|c|}{ Model 1 = Unadjusted. } \\
\hline \multicolumn{7}{|c|}{ Model 2 = Model $1+$ Age, Sex and body mass index (BMI). } \\
\hline \multicolumn{7}{|c|}{$\begin{array}{l}\text { Model } 3=\text { Model } 2+\text { Familyhistory } 2 \text { (family history of cardiovascular disease), TC (total cholesterol), } \\
\text { CREA (creatinine) }\end{array}$} \\
\hline
\end{tabular}

Total leukocyte count revealed significant correlation which remained still after adjustment in MetS, hypertension, and T2DM groups for subjects aged 40-64-year-old (Table 4). In the above three diseasespecific groups, monocyte count was significant originally but failed in hypertension group with Model 3 adjustment. 
Table 4

Multivariate hazard ratios of future metabolic syndrome, hypertension and type 2 diabetes mellitus by white blood cell count and differential. (Age $=40-64$ )

\begin{tabular}{|c|c|c|c|c|c|c|}
\hline \multirow[t]{3}{*}{ Variables } & \multicolumn{6}{|l|}{ MetS } \\
\hline & \multicolumn{2}{|l|}{ Model 1} & \multicolumn{2}{|l|}{ Model 2} & \multicolumn{2}{|l|}{ Model 3} \\
\hline & $\operatorname{Exp}(\beta)(95 \% \mathrm{Cl})$ & $P$ value & $\operatorname{Exp}(\beta)(95 \% \mathrm{Cl})$ & $P$ value & $\operatorname{Exp}(\beta)(95 \% \mathrm{Cl})$ & $P$ value \\
\hline WBC & $\begin{array}{l}1.215(1.157 \\
1.277)\end{array}$ & $<.001^{\star *}$ & $\begin{array}{l}1.133(1.072, \\
1.197)\end{array}$ & $<.001^{\star \star}$ & $\begin{array}{l}1.143(1.045 \\
1.249)\end{array}$ & $0.003^{* *}$ \\
\hline NEUT & $\begin{array}{l}1.012(0.998 \\
1.025)\end{array}$ & 0.091 & $\begin{array}{l}1.017(1.003 \\
1.031)\end{array}$ & $0.020 *$ & $\begin{array}{l}1.010(0.988 \\
1.033)\end{array}$ & 0.360 \\
\hline LYMP & $\begin{array}{l}0.991(0.976 \\
1.005)\end{array}$ & 0.208 & $\begin{array}{l}0.991(0.976 \\
1.006)\end{array}$ & 0.230 & $\begin{array}{l}1.002(0.978 \\
1.025)\end{array}$ & 0.896 \\
\hline MONO & $\begin{array}{l}0.890(0.833 \\
0.951)\end{array}$ & $0.001^{* \star}$ & $\begin{array}{l}0.858(0.802 \\
0.919)\end{array}$ & $<.001^{\star \star}$ & $\begin{array}{l}0.901(0.817 \\
0.993)\end{array}$ & $0.036^{\star}$ \\
\hline EOSIN & $\begin{array}{l}1.027(0.978 \\
1.079)\end{array}$ & 0.283 & $\begin{array}{l}0.980(0.927 \\
1.036)\end{array}$ & 0.476 & $\begin{array}{l}0.924(0.844 \\
1.011)\end{array}$ & 0.085 \\
\hline BAS & $\begin{array}{l}0.864(0.630 \\
1.183)\end{array}$ & 0.361 & $\begin{array}{l}0.910(0.671 \\
1.235)\end{array}$ & 0.545 & $\begin{array}{l}0.817(0.530 \\
1.261)\end{array}$ & 0.362 \\
\hline \multirow[t]{3}{*}{ Variables } & \multicolumn{6}{|l|}{ HTN } \\
\hline & \multicolumn{2}{|l|}{ Model 1} & \multicolumn{2}{|l|}{ Model 2} & \multicolumn{2}{|l|}{ Model 3} \\
\hline & $\operatorname{Exp}(\beta)(95 \% \mathrm{Cl})$ & $P$ value & $\operatorname{Exp}(\beta)(95 \% \mathrm{Cl})$ & $P$ value & $\operatorname{Exp}(\beta)(95 \% \mathrm{Cl})$ & $P$ value \\
\hline WBC & $\begin{array}{l}1.121(1.073, \\
1.172)\end{array}$ & $\begin{array}{l}<.001^{* *} \\
\end{array}$ & $\begin{array}{l}1.072(1.020 \\
1.126)\end{array}$ & 0.006 ** & $\begin{array}{l}1.105(1.024 \\
1.193)\end{array}$ & $0.010^{\star}$ \\
\hline NEUT & $\begin{array}{l}1.021(1.010 \\
1.032)\end{array}$ & $<.001^{<\star *}$ & $\begin{array}{l}1.027(1.016 \\
1.039)\end{array}$ & $<.001^{\star \star \star}$ & $\begin{array}{l}1.028(1.010 \\
1.045)\end{array}$ & $0.002^{\star \star}$ \\
\hline
\end{tabular}

${ }^{\star} \mathrm{p}<0.05,{ }^{*} \mathrm{p}<0.01$

Abbreviation:

MetS, metabolic syndrome; HTN, hypertension; DM, diabetes mellitus; WBC, white blood cell count; NEUT, neutrophil count; LYMP, lymphocyte count; MONO, monocyte count; EOSIN, eosinophil count; BAS, basophil count

Adjusted covariates:

Model 1 = Unadjusted.

Model 2 = Model $1+$ Age, Sex and body mass index (BMI).

Model 3 = Model 2 + Familyhistory2 (family history of cardiovascular disease), TC (total cholesterol), CREA (creatinine) 


\begin{tabular}{|c|c|c|c|c|c|c|}
\hline \multirow[t]{3}{*}{ Variables } & \multicolumn{6}{|l|}{ MetS } \\
\hline & \multicolumn{2}{|l|}{ Model 1} & \multicolumn{2}{|l|}{ Model 2} & \multicolumn{2}{|l|}{ Model 3} \\
\hline & $\operatorname{Exp}(\beta)(95 \% \mathrm{Cl})$ & $P$ value & $\operatorname{Exp}(\beta)(95 \% \mathrm{Cl})$ & $P$ value & $\operatorname{Exp}(\beta)(95 \% \mathrm{Cl})$ & $P$ value \\
\hline LYMP & $\begin{array}{l}0.981(0.969 \\
0.992)\end{array}$ & 0.001 ** & $\begin{array}{l}0.978(0.967 \\
0.990)\end{array}$ & $<.001^{\star \star}$ & $\begin{array}{l}0.979(0.961 \\
0.997)\end{array}$ & $0.024^{\star}$ \\
\hline MONO & $\begin{array}{l}0.925(0.878 \\
0.974)\end{array}$ & $0.003^{\star \star}$ & $\begin{array}{l}0.909 \text { ( } 0.863 \\
0.959)\end{array}$ & $<.001 * \star$ & $\begin{array}{l}0.947(0.880 \\
1.019)\end{array}$ & 0.144 \\
\hline EOSIN & $\begin{array}{l}0.993(0.951, \\
1.037)\end{array}$ & 0.751 & $\begin{array}{l}0.962(0.918 \\
1.007)\end{array}$ & 0.099 & $\begin{array}{l}0.927(0.865 \\
0.994)\end{array}$ & $0.032^{\star}$ \\
\hline BAS & $\begin{array}{l}0.713(0.539 \\
0.943)\end{array}$ & $0.018^{*}$ & $\begin{array}{l}0.724(0.552, \\
0.950)\end{array}$ & $0.020^{*}$ & $\begin{array}{l}0.647(0.440 \\
0.950)\end{array}$ & $0.026^{\star}$ \\
\hline \multirow[t]{3}{*}{ Variables } & \multicolumn{6}{|l|}{ Type 2 DM } \\
\hline & \multicolumn{2}{|l|}{ Model 1} & \multicolumn{2}{|l|}{ Model 2} & \multicolumn{2}{|l|}{ Model 3} \\
\hline & $\operatorname{Exp}(\beta)(95 \% \mathrm{Cl})$ & $P$ value & $\operatorname{Exp}(\beta)(95 \% \mathrm{Cl})$ & $P$ value & $\operatorname{Exp}(\beta)(95 \% \mathrm{Cl})$ & $P$ value \\
\hline WBC & $\begin{array}{l}1.213(1.128 \\
1.306)\end{array}$ & $<.001^{<*}$ & $\begin{array}{l}1.244(1.150 \\
1.345)\end{array}$ & $<.001^{\star \star}$ & $\begin{array}{l}1.254(1.113 \\
1.412)\end{array}$ & $\hat{0}_{0.001 * *}$ \\
\hline NEUT & $\begin{array}{l}1.029(1.008 \\
1.050)\end{array}$ & $0.005^{\star \star}$ & $\begin{array}{l}1.037(1.015 \\
1.059)\end{array}$ & $0.001^{* *}$ & $\begin{array}{l}1.028(0.999 \\
1.057)\end{array}$ & 0.055 \\
\hline LYMP & $\begin{array}{l}0.972 \text { (0.951, } \\
0.994)\end{array}$ & $0.013^{*}$ & $\begin{array}{l}0.967(0.945 \\
0.990)\end{array}$ & $0.005^{\star \star}$ & $\begin{array}{l}0.977 \text { (0.947, } \\
1.008)\end{array}$ & 0.147 \\
\hline MONO & $\begin{array}{l}0.841(0.760 \\
0.930)\end{array}$ & $0.001^{\star *}$ & $\begin{array}{l}0.844(0.762 \\
0.934)\end{array}$ & $0.001^{\star *}$ & $\begin{array}{l}0.864(0.761 \\
0.981)\end{array}$ & $0.024^{\star}$ \\
\hline EOSIN & $\begin{array}{l}1.036(0.962, \\
1.115)\end{array}$ & 0.350 & $\begin{array}{l}1.003(0.927 \\
1.084)\end{array}$ & 0.944 & $\begin{array}{l}1.004 \text { (0.909, } \\
1.109)\end{array}$ & 0.937 \\
\hline
\end{tabular}

* $p<0.05, * * p<0.01$

Abbreviation:

MetS, metabolic syndrome; HTN, hypertension; DM, diabetes mellitus; WBC, white blood cell count; NEUT, neutrophil count; LYMP, lymphocyte count; MONO, monocyte count; EOSIN, eosinophil count; BAS, basophil count

Adjusted covariates:

Model 1 = Unadjusted.

Model 2 = Model $1+$ Age, Sex and body mass index (BMI).

Model 3 = Model 2 + Familyhistory2 (family history of cardiovascular disease), TC (total cholesterol), CREA (creatinine) 


\begin{tabular}{|c|c|c|c|c|c|c|}
\hline \multirow[t]{3}{*}{ Variables } & \multicolumn{6}{|l|}{ MetS } \\
\hline & \multicolumn{2}{|l|}{ Model 1} & \multicolumn{2}{|l|}{ Model 2} & \multicolumn{2}{|l|}{ Model 3} \\
\hline & $\operatorname{Exp}(\beta)(95 \% \mathrm{Cl})$ & $P$ value & $\operatorname{Exp}(\beta)(95 \% \mathrm{Cl})$ & $P$ value & $\operatorname{Exp}(\beta)(95 \% \mathrm{Cl})$ & $P$ value \\
\hline BAS & $\begin{array}{l}0.526(0.289 \\
0.959)\end{array}$ & $0.036^{\star}$ & $\begin{array}{l}0.554(0.315 \\
0.976)\end{array}$ & $0.041^{*}$ & $\begin{array}{l}0.745(0.410 \\
1.353)\end{array}$ & 0.333 \\
\hline \multicolumn{7}{|c|}{${ }^{*} \mathrm{p}<0.05, * * \mathrm{p}<0.01$} \\
\hline \multicolumn{7}{|c|}{ Abbreviation: } \\
\hline \multicolumn{7}{|c|}{$\begin{array}{l}\text { MetS, metabolic syndrome; HTN, hypertension; DM, diabetes mellitus; WBC, white blood cell count; } \\
\text { NEUT, neutrophil count; LYMP, Iymphocyte count; MONO, monocyte count; EOSIN, eosinophil count; } \\
\text { BAS, basophil count }\end{array}$} \\
\hline \multicolumn{7}{|c|}{ Adjusted covariates: } \\
\hline \multicolumn{7}{|c|}{ Model 1 = Unadjusted. } \\
\hline \multicolumn{7}{|c|}{ Model $2=$ Model $1+$ Age, Sex and body mass index (BMI). } \\
\hline \multicolumn{7}{|c|}{$\begin{array}{l}\text { Model } 3=\text { Model } 2+\text { Familyhistory } 2 \text { (family history of cardiovascular disease), TC (total cholesterol), } \\
\text { CREA (creatinine) }\end{array}$} \\
\hline
\end{tabular}

In Table 5, significant association was only demonstrated between total leukocyte count and the three disease-specific groups in Model 1 for subjects aged above 65-year-old. 
Table 5

Multivariate hazard ratios of future metabolic syndrome, hypertension and type 2 diabetes mellitus by white blood cell count and differential. (Age > 65)

\begin{tabular}{|c|c|c|c|c|c|c|}
\hline \multirow[t]{3}{*}{ Variables } & \multicolumn{6}{|l|}{ MetS } \\
\hline & \multicolumn{2}{|l|}{ Model 1} & \multicolumn{2}{|l|}{ Model 2} & \multicolumn{2}{|l|}{ Model 3} \\
\hline & $\operatorname{Exp}(\beta)(95 \% \mathrm{Cl})$ & $P$ value & $\operatorname{Exp}(\beta)(95 \% \mathrm{Cl})$ & $\begin{array}{l}P \\
\text { value }\end{array}$ & $\operatorname{Exp}(\beta)(95 \% \mathrm{Cl})$ & $\begin{array}{l}P \\
\text { value }\end{array}$ \\
\hline WBC & $\begin{array}{l}1.267(1.178, \\
1.363)\end{array}$ & $<.001^{\star * *}$ & $\begin{array}{l}1.059(0.922, \\
1.216)\end{array}$ & 0.418 & $\begin{array}{l}1.078(0.917 \\
1.268)\end{array}$ & 0.362 \\
\hline NEUT & $\begin{array}{l}1.044 \text { (1.001, } \\
1.089)\end{array}$ & $0.044^{*}$ & $\begin{array}{l}1.044 \text { (0.996, } \\
1.094)\end{array}$ & 0.070 & $\begin{array}{l}0.991(0.930 \\
1.056)\end{array}$ & 0.780 \\
\hline LYMP & $\begin{array}{l}0.959(0.918 \\
1.002)\end{array}$ & 0.063 & $\begin{array}{l}0.958(0.910 \\
1.008)\end{array}$ & 0.096 & $\begin{array}{l}1.022(0.952 \\
1.096)\end{array}$ & 0.551 \\
\hline MONO & $\begin{array}{l}0.920(0.750 \\
1.128)\end{array}$ & 0.421 & $\begin{array}{l}0.854(0.677 \\
1.077)\end{array}$ & 0.181 & $\begin{array}{l}0.890(0.636 \\
1.246)\end{array}$ & 0.497 \\
\hline EOSIN & $\begin{array}{l}0.966(0.803 \\
1.162)\end{array}$ & 0.716 & $\begin{array}{l}0.985(0.802 \\
1.211)\end{array}$ & 0.888 & $\begin{array}{l}0.926(0.673 \\
1.275)\end{array}$ & 0.638 \\
\hline BAS & $\begin{array}{l}0.802(0.234 \\
2.748)\end{array}$ & 0.725 & $\begin{array}{l}0.882(0.221 \\
3.525)\end{array}$ & 0.859 & $\begin{array}{l}0.573(0.081 \\
4.058)\end{array}$ & 0.577 \\
\hline \multirow[t]{3}{*}{ Variables } & \multicolumn{6}{|l|}{ HTN } \\
\hline & \multicolumn{2}{|l|}{ Model 1} & \multicolumn{2}{|l|}{ Model 2} & \multicolumn{2}{|l|}{ Model 3} \\
\hline & $\operatorname{Exp}(\beta)(95 \% \mathrm{Cl})$ & $P$ value & $\operatorname{Exp}(\beta)(95 \% \mathrm{Cl})$ & $\begin{array}{l}P \\
\text { value }\end{array}$ & $\operatorname{Exp}(\beta)(95 \% \mathrm{Cl})$ & $\begin{array}{l}\mathrm{P} \\
\text { value }\end{array}$ \\
\hline WBC & $\begin{array}{l}1.129(1.055 \\
1.209)\end{array}$ & $<.001^{* *}$ & $\begin{array}{l}1.043(0.931, \\
1.169)\end{array}$ & 0.469 & $\begin{array}{l}1.121(0.986 \\
1.274)\end{array}$ & 0.082 \\
\hline NEUT & $\begin{array}{l}1.017(0.980 \\
1.056)\end{array}$ & 0.369 & $\begin{array}{l}1.017(0.978 \\
1.057)\end{array}$ & 0.396 & $\begin{array}{l}1.025(0.978 \\
1.074)\end{array}$ & 0.307 \\
\hline \multicolumn{7}{|c|}{${ }^{*} p<0.05, * \star p<0.01$} \\
\hline \multicolumn{7}{|c|}{ Abbreviation: } \\
\hline \multicolumn{7}{|c|}{$\begin{array}{l}\text { MetS, metabolic syndrome; HTN, hypertension; DM, diabetes mellitus; WBC, white blood cell count; } \\
\text { NEUT, neutrophil count; LYMP, lymphocyte count; MONO, monocyte count; EOSIN, eosinophil count; } \\
\text { BAS, basophil count }\end{array}$} \\
\hline \multicolumn{7}{|c|}{ Adjusted covariates: } \\
\hline \multicolumn{7}{|c|}{ Model 1 = Unadjusted. } \\
\hline \multicolumn{7}{|c|}{ Model 2 = Model $1+$ Age, Sex and body mass index (BMI). } \\
\hline $\begin{array}{l}\text { Model } 3= \\
\text { CREA (cre }\end{array}$ & $\begin{array}{l}\text { lodel } 2+\text { Familyhis } \\
\text { inine) }\end{array}$ & y2 (famil & history of cardiov & ular dis & ase), TC (total ch & terol), \\
\hline
\end{tabular}




\begin{tabular}{|c|c|c|c|c|c|c|}
\hline \multirow[t]{3}{*}{ Variables } & \multicolumn{6}{|l|}{ MetS } \\
\hline & \multicolumn{2}{|l|}{ Model 1} & \multicolumn{2}{|l|}{ Model 2} & \multicolumn{2}{|l|}{ Model 3} \\
\hline & $\operatorname{Exp}(\beta)(95 \% \mathrm{Cl})$ & $P$ value & $\operatorname{Exp}(\beta)(95 \% \mathrm{Cl})$ & $\begin{array}{l}P \\
\text { value }\end{array}$ & $\operatorname{Exp}(\beta)(95 \% \mathrm{Cl})$ & $\begin{array}{l}P \\
\text { value }\end{array}$ \\
\hline LYMP & $\begin{array}{l}0.980(0.942 \\
1.019)\end{array}$ & 0.308 & $\begin{array}{l}0.988(0.947 \\
1.031)\end{array}$ & 0.582 & $\begin{array}{l}0.987(0.938 \\
1.038)\end{array}$ & 0.602 \\
\hline MONO & $\begin{array}{l}0.953(0.805 \\
1.130)\end{array}$ & 0.581 & $\begin{array}{l}0.888(0.730 \\
1.079)\end{array}$ & 0.232 & $\begin{array}{l}0.926(0.736 \\
1.166)\end{array}$ & 0.514 \\
\hline EOSIN & $\begin{array}{l}1.038(0.921, \\
1.170)\end{array}$ & 0.542 & $\begin{array}{l}0.973(0.842, \\
1.126)\end{array}$ & 0.715 & $\begin{array}{l}0.900(0.747 \\
1.085)\end{array}$ & 0.268 \\
\hline BAS & $\begin{array}{l}0.542(0.172 \\
1.706)\end{array}$ & 0.295 & $\begin{array}{l}0.428(0.124 \\
1.480)\end{array}$ & 0.180 & $\begin{array}{l}0.149(0.029 \\
0.754)\end{array}$ & $0.021 *$ \\
\hline \multirow[t]{3}{*}{ Variables } & \multicolumn{6}{|l|}{ Type 2 DM } \\
\hline & \multicolumn{2}{|l|}{ Model 1} & \multicolumn{2}{|l|}{ Model 2} & \multicolumn{2}{|l|}{ Model 3} \\
\hline & $\operatorname{Exp}(\beta)(95 \% \mathrm{Cl})$ & $P$ value & $\operatorname{Exp}(\beta)(95 \% \mathrm{Cl})$ & $\begin{array}{l}P \\
\text { value }\end{array}$ & $\operatorname{Exp}(\beta)(95 \% \mathrm{Cl})$ & $\begin{array}{l}P \\
\text { value }\end{array}$ \\
\hline WBC & $\begin{array}{l}1.288(1.171, \\
1.418)\end{array}$ & $<.001^{\star *}$ & $\begin{array}{l}1.140(0.969 \\
1.341)\end{array}$ & 0.113 & $\begin{array}{l}1.093(0.902 \\
1.325)\end{array}$ & 0.363 \\
\hline NEUT & $\begin{array}{l}1.033(0.974, \\
1.095)\end{array}$ & 0.276 & $\begin{array}{l}1.031(0.968 \\
1.099)\end{array}$ & 0.342 & $\begin{array}{l}1.016(0.936 \\
1.103)\end{array}$ & 0.706 \\
\hline LYMP & $\begin{array}{l}0.960(0.901 \\
1.022)\end{array}$ & 0.197 & $\begin{array}{l}0.964(0.897 \\
1.035)\end{array}$ & 0.306 & $\begin{array}{l}0.985(0.897 \\
1.081)\end{array}$ & 0.753 \\
\hline MONO & $\begin{array}{l}0.982(0.752, \\
1.283)\end{array}$ & 0.897 & $\begin{array}{l}0.867(0.626 \\
1.202)\end{array}$ & 0.392 & $\begin{array}{l}0.938(0.622 \\
1.415)\end{array}$ & 0.760 \\
\hline EOSIN & $\begin{array}{l}1.038(0.843 \\
1.277)\end{array}$ & 0.728 & $\begin{array}{l}1.059(0.823 \\
1.363)\end{array}$ & 0.654 & $\begin{array}{l}0.970(0.673 \\
1.400)\end{array}$ & 0.872 \\
\hline \multicolumn{7}{|c|}{${ }^{\star} \mathrm{p}<0.05,{ }^{*} \mathrm{p}<0.01$} \\
\hline \multicolumn{7}{|c|}{ Abbreviation: } \\
\hline \multicolumn{7}{|c|}{$\begin{array}{l}\text { MetS, metabolic syndrome; HTN, hypertension; DM, diabetes mellitus; WBC, white blood cell count; } \\
\text { NEUT, neutrophil count; LYMP, lymphocyte count; MONO, monocyte count; EOSIN, eosinophil count; } \\
\text { BAS, basophil count }\end{array}$} \\
\hline \multicolumn{7}{|c|}{ Adjusted covariates: } \\
\hline \multicolumn{7}{|c|}{ Model 1 = Unadjusted. } \\
\hline \multicolumn{7}{|c|}{ Model 2 = Model $1+$ Age, Sex and body mass index (BMI). } \\
\hline $\begin{array}{l}\text { Model } 3= \\
\text { CREA (crea }\end{array}$ & $\begin{array}{l}\text { odel } 2 \text { + Familyhi } \\
\text { inine) }\end{array}$ & y2 (famil & nistory of cardio & ular dis & se), TC (total ch & erol), \\
\hline
\end{tabular}




\begin{tabular}{|c|c|c|c|c|c|c|}
\hline \multirow[t]{3}{*}{ Variables } & \multicolumn{6}{|l|}{ MetS } \\
\hline & \multicolumn{2}{|l|}{ Model 1} & \multicolumn{2}{|l|}{ Model 2} & \multicolumn{2}{|l|}{ Model 3} \\
\hline & $\operatorname{Exp}(\beta)(95 \% \mathrm{Cl})$ & $P$ value & $\operatorname{Exp}(\beta)(95 \% \mathrm{Cl})$ & $\begin{array}{l}P \\
\text { value }\end{array}$ & $\operatorname{Exp}(\beta)(95 \% \mathrm{Cl})$ & $\begin{array}{l}P \\
\text { value }\end{array}$ \\
\hline BAS & $\begin{array}{l}3.158(0.657 \\
15.179)\end{array}$ & 0.151 & $\begin{array}{l}2.814(0.442 \\
17.903)\end{array}$ & 0.273 & $\begin{array}{l}1.015(0.099 \\
10.431)\end{array}$ & 0.990 \\
\hline \multicolumn{7}{|c|}{${ }^{*} p<0.05,{ }^{* *} p<0.01$} \\
\hline \multicolumn{7}{|c|}{ Abbreviation: } \\
\hline \multicolumn{7}{|c|}{$\begin{array}{l}\text { MetS, metabolic syndrome; HTN, hypertension; DM, diabetes mellitus; WBC, white blood cell count; } \\
\text { NEUT, neutrophil count; LYMP, Iymphocyte count; MONO, monocyte count; EOSIN, eosinophil count; } \\
\text { BAS, basophil count }\end{array}$} \\
\hline \multicolumn{7}{|c|}{ Adjusted covariates: } \\
\hline \multicolumn{7}{|c|}{ Model 1 = Unadjusted. } \\
\hline \multicolumn{7}{|c|}{ Model 2 = Model $1+$ Age, Sex and body mass index (BMI). } \\
\hline \multicolumn{7}{|c|}{$\begin{array}{l}\text { Model } 3 \text { = Model } 2+\text { Familyhistory2 (family history of cardiovascular disease), TC (total cholesterol), } \\
\text { CREA (creatinine) }\end{array}$} \\
\hline
\end{tabular}

\section{Discussion}

The aim of the present study was to investigate the possible contribution of leukocyte subgroups to future hypertension, T2DM, and MetS as well as its components in Taiwan citizens. This study also demonstrated a potential age-dependent association between total leukocyte count, monocyte count and risk of developing the three diseases mentioned above. Our results revealed that total leukocyte count and monocyte count were positively correlated with future MetS, hypertension, and T2DM in middle-aged participants. In addition, the significant association between total leukocyte count and monocyte count with MetS components had been identified.

In the present study, the significant association between the total leucocyte, neutrophil and lymphocyte with MetS was consistent with previous studies with a smaller cohort of participants $(1,17,18)$. Furthermore, our study indicated the fact that elevated total leukocyte count was significantly and positively correlated with WC, SBP, DBP, fasting glucose, and TG while negatively correlated with HDL-C. The above relationship supported by previous longitudinal study (19) had demonstrated the important role for total leukocyte count playing in MetS components.

Multiple risk factors contributing to CVD such as diabetes, obesity, hypertension, and dyslipidemia has been well established (20). It is well established that the prevalence of MetS, hypertension and T2DM all rise from young to old ages (21-24). As far as the author reviewed, there were few reports focusing on the relationship between total/subtype leukocyte counts and risk of future MetS, hypertension, and T2DM 
by age distribution. Both studies done by Nakanishi et al and Nilsson et al $(25,26)$ revealed the positive correlation of total leukocyte count in adult age for MetS. However, this trend could only be identified in the subjects categorized into young-old group ( $\geqq 65$ and $<75$ ) then disappear in older age subjects according to Chuang et al, 2015 (1). Our results also demonstrated similar evidence that total leukocyte count showed no significant correlation in both multivariate models of older age subjects.

The biological mechanisms for higher total leukocyte count causing increasing risk for MetS might be explained through high inflammatory activities. White blood cell, high- sensitivity C-reactive protein (hsCRP), interleukin-6 (IL-6), and tumor necrosis factor-alpha (TNF-a) levels were all significantly elevated in the MetS group according to previous research (27). Produced by activated macrophages within adipose tissue, aforementioned inflammatory cytokines, especially TNF-a, takes an important part in obesitylinked insulin resistance (28). Composed of enlarged dead adipocytes surrounded by numerous activated macrophages, these crown-like structures had been recognized as characteristic histological pattern for dysfunction of adipose tissue(29). Moreover, as a possible systemic manifestation of adipose tissue dysfunction, MetS had been prooved to play a key role in lots of pathophysiological mechanisms including insulin resistance, systemic inflammation, and endothelial dysfunction $(30,31)$.

In the present study, we identified that elevated total leukocyte count with the possibility of predicting future T2DM for both young and middle-aged subjects, which was consistent with previous research (1, $32,33)$. For the close relation between MetS and T2DM, it is reasonable to discover these positive associations.

Considered as a chronic inflammatory process, atherosclerosis centers in the pathophysiological pathway leading to hypertension $(34,35)$. Serving as a biomarker of chronic inflammation, it is not surprising that total leukocyte count showed positive association with hypertension in prior research, which was all in line with our results $(36,37)$.

As far as we know, this research is the first to investigate the role for monocyte count playing in predicting future MetS, hypertension, and T2DM according to age distribution. Analogous to total leukocyte count, monocyte count holds prospective associations with the above three diseases in middle-aged participants. While for young subjects, the aforementioned association between monocyte count and future T2DM vanished.

By means of either acting directly on macrophages to shift polarization or prime human monocyte differentiation into anti-inflammatory M2 macrophages for healthy individual, Adiponectin is an adipokine with multiple effects on cytokine secretion, metabolism of lipids, and direct insulin sensitizing activity $(38,39)$. As a cluster of risk factors for CVD and diabetes, MetS is well considered to be a proinflammatory state (36). Infiltration of macrophage and lymphocyte in adipose tissue may hold the key to pathogenesis of obesity-mediated metabolic disorders through crosstalk and release of various biomediators that promote inflammation (40). Increased systemic oxidative stress derived from MetS is associated with adiponectin deficiency, which drives monocytes toward M1 rather than M2 phenotype of macrophages in the circulation of MetS subjects $(41,42)$. 
Dysregulation of M1 and M2 phenotype of macrophages as well as increasing amounts of circulating monocytes also exist in T2DM subjects $(43,44)$. Previous studies demonstrated an acute proceeding cytokine-mediated response initiated by both the innate and adaptive immune system which comes along with T2DM. In the tissues where they are generated, cytokines such as IL-6, IL-1 the capability of promoting insulin resistance and then influence other locations through circulation including cardiac and skeletal muscle as well as endothelium of vascular walls $(45,46)$.

An article published in the Cellular and Molecular Life Sciences in 2016 reported a novel concept about "trained immunity" in patients with diabetes, a theory that long-term functional reprogramming of monocytes and macrophages, result from hyperglycemia, centers in the phenomenon of hyperglycemic memory leading to atherosclerotic plaques development and subsequent macrovascular complications (47).

Localizing to district of injury, infection or stress, innate immune cells serve as part of the inflammatory process through reacting to chemokine and adhesion molecules such as monocyte chemoattractant protein-1 (MCP-1) and vascular cell adhesion molecule-1 (VCAM-1). It is still uncertain about how monocyte promote hypertension, a consequence of low-grade inflammation. The possible pathophysiology relationship between monocytes and hypertension can be summarized into the following: (1) expression of functional Angiotensin II and mineralocorticoid receptors on monocytes leads to production of reactive oxygen species (ROS) via activation of reduced nicotinamide adenine dinucleotide (NADPH) oxidase; (2) tissue damage via the CC chemokine receptor 2 (CCR2) expressed on monocytes, which results from chemotaxis of monocytes and macrophages by increased MCP-1 (48).

The main strength of the present study is that this is the first longitudinal study to identify the value of monocyte count as predictors for future MetS, hypertension, and T2DM in different age subgroups. Moreover, the role of total leukocyte count on the risk of MetS and its components as well as development of the aforementioned diseases were also demonstrated. Nevertheless, several limitations still existed in the present research. First, the participants of our study were not from a general population, but from a health promotion center located in the capital city of Taiwan, which means they may fail to represent the general population for relatively higher socio-economic status. Second, insulin resistance, as the central concept of MetS, was not measured through glucose tolerance test in the current research. Third, cytokines (such as IL-6 and TNF-a) were not measured in this research on account of budget shortage. Fourth, our study lacked information on drug use such as antidiabetic, anti-hypertensive, or statin medication, which could affect hematological parameters or metabolism, should be recorded in specific details for risk stratification in further research. At last, we did not gain available data of cardiovascular event or all-cause mortality, which might cause considerable interference in the interpretation of our results. Future studies with plasma insulin levels, circulating levels of cytokines, detailed record of medication usage and incidence of all-cause mortality and/or cardiovascular events would provide further aid and insights to our current discoveries. 
In conclusion, our results explore the promising value of total leukocyte count and monocyte count to recognize the high-risk subjects, especially meddle-aged ones, having MetS, hypertension, and T2DM in the present or the future. Elevated levels of these easily measured hematologic parameters might take crucial parts in these modern diseases, which special attention should be paid by healthcare providers, from the present into the future.

\section{Abbreviations List}

BMl, body mass index;

BUN, blood urea nitrogen;

CREA, creatinine;

CVD, cardiovascular disease;

DBP, diastolic blood pressure;

EDTA, Ethylenediaminetetraacetic acid;

HDL-C, high-density lipoprotein cholesterol;

IL-1 $\beta$, interleukin-1 $\beta$;

IL-6, interleukin-6;

LDL-C, low-density lipoprotein cholesterol;

MCP- 1, monocyte chemoattractant protein-1;

MetS, metabolic syndrome;

SBP, systolic blood pressure;

TC, total cholesterol;

T2DM, type 2 diabetes mellitus;

TG, triacylglycerol;

TNF-a, tumor necrosis factor-alpha;

UA, uric acid;

WC, waist circumference 


\section{Declarations}

\section{Acknowledgments}

The authors would like to acknowledge Dr. Li-Wei Wu for his contributions to data collection and his crucial review of this manuscript.

\section{Author contribution statement}

Yen-Wei Li contributed to the design of the study, was responsible for the management and retrieval of data, contributed to initial data analysis and interpretation, and drafted the initial manuscript. Yen-Wei Li, Tung-Wei Kao, Chao-Yin Kuo, Pi-Kai Chang, Wei-Liang Chen, Li-Wei Wu decided upon the data collection methods. Yen-Wei Li and Li-Wei Wu were also responsible for the data analysis decisions. Li-Wei Wu conceptualized and designed the study, supervised all aspects of the study, critically reviewed and revised the manuscript, and approved the final manuscript as submitted. All authors meet the ICMJE criteria for authorship.

\section{Declarations of interest}

The authors declare that they have no conflict of interests.

\section{Funding information}

This work was supported by a grant to Li-Wei Wu [TSGH-C104-PH-2, TSGH-PH-105-2, TSGH-PH-106-3, TSGH-PH-107-4, TSGH-C108-050, TSGH-E-109223, MAB-105-031, MAB-106-037, MAB-106-039, MAB-107043, MAB-108-069, MAB-109-077]. All authors declare that they have no conflicts of interest.

\section{References}

1. Chuang T-J, Liu J-S, Li P-F, Chang Hw, Hsieh C-H, Chang J-B, et al. The relationships between hematogram and metabolic syndrome in elderly subgroups: A Taiwan cohort study. Archives of Gerontology and Geriatrics. 2016;63:59-66.

2. Reaven GM. Banting lecture 1988. Role of insulin resistance in human disease. Diabetes. 1988;37(12):1595-607.

3. Pearson TA, Mensah GA, Alexander RW, Anderson JL, Cannon RO, 3rd, Criqui M, et al. Markers of inflammation and cardiovascular disease: application to clinical and public health practice: A statement for healthcare professionals from the Centers for Disease Control and Prevention and the American Heart Association. Circulation. 2003;107(3):499-511.

4. Wang YY, Lin SY, Liu PH, Cheung BM, Lai WA. Association between hematological parameters and metabolic syndrome components in a Chinese population. Journal of diabetes and its complications. 2004;18(6):322-7. 
5. Scherer PE. Adipose tissue: from lipid storage compartment to endocrine organ. Diabetes. 2006;55(6):1537-45.

6. Olefsky JM, Glass CK. Macrophages, inflammation, and insulin resistance. Annual review of physiology. 2010;72:219-46.

7. Nagasawa N, Tamakoshi K, Yatsuya H, Hori Y, Ishikawa M, Murata C, et al. Association of white blood cell count and clustered components of metabolic syndrome in Japanese men. Circulation journal : official journal of the Japanese Circulation Society. 2004;68(10):892-7.

8. Ohshita K, Yamane K, Hanafusa M, Mori H, Mito K, Okubo M, et al. Elevated white blood cell count in subjects with impaired glucose tolerance. Diabetes Care. 2004;27(2):491-6.

9. Tong PC, Lee KF, So WY, Ng MH, Chan WB, Lo MK, et al. White blood cell count is associated with macro- and microvascular complications in chinese patients with type 2 diabetes. Diabetes Care. 2004;27(1):216-22.

10. Kannel WB, Anderson K, Wilson PW. White blood cell count and cardiovascular disease. Insights from the Framingham Study. Jama. 1992;267(9):1253-6.

11. Fadini GP, Marcuzzo G, Marescotti MC, de Kreutzenberg SV, Avogaro A. Elevated white blood cell count is associated with prevalence and development of the metabolic syndrome and its components in the general population. Acta diabetologica. 2012;49(6):445-51.

12. Odagiri K, Uehara A, Mizuta I, Yamamoto M, Kurata C. Longitudinal study on white blood cell count and the incidence of metabolic syndrome. Internal medicine (Tokyo, Japan). 2011;50(21):2491-8.

13. Dalton M, Cameron AJ, Zimmet PZ, Shaw JE, Jolley D, Dunstan DW, et al. Waist circumference, waisthip ratio and body mass index and their correlation with cardiovascular disease risk factors in Australian adults. J Intern Med. 2003;254(6):555-63.

14. Richmond W. Preparation and Properties of a Cholesterol Oxidase from Nocardia sp. and Its Application to the Enzymatic Assay of Total Cholesterol in Serum. Clinical Chemistry. 1973;19(12):1350-6.

15. Warnick GR, Benderson J, Albers JJ. Dextran sulfate-Mg2+ precipitation procedure for quantitation of high-density-lipoprotein cholesterol. Clinical Chemistry. 1982;28(6):1379-88.

16. Alberti KG, Zimmet P, Shaw J. Metabolic syndrome--a new world-wide definition. A Consensus Statement from the International Diabetes Federation. Diabetic medicine : a journal of the British Diabetic Association. 2006;23(5):469-80.

17. Tanigawa T, Iso H, Yamagishi K, Muraki I, Kawamura N, Nakata A, et al. Association of lymphocyte sub-populations with clustered features of metabolic syndrome in middle-aged Japanese men. Atherosclerosis.173(2):295-300.

18. Lao XQ, Neil Thomas G, Jiang C, Zhang W, Adab P, Lam TH, et al. White blood cell count and the metabolic syndrome in older Chinese: The Guangzhou Biobank Cohort Study. Atherosclerosis. 2008;201(2):418-24.

19. Pei C, Chang J-B, Hsieh C-H, Lin J-D, Hsu C-H, Pei D, et al. Using white blood cell counts to predict metabolic syndrome in the elderly: A combined cross-sectional and longitudinal study. European 
Journal of Internal Medicine.26(5):324-9.

20. Quiat D, Olson EN. MicroRNAs in cardiovascular disease: from pathogenesis to prevention and treatment. The Journal of clinical investigation. 2013;123(1):11-8.

21. Buford TW. Hypertension and aging. Ageing research reviews. 2016;26:96-111.

22. Kalyani RR, Egan JM. Diabetes and altered glucose metabolism with aging. Endocrinology and metabolism clinics of North America. 2013;42(2):333-47.

23. Cameron AJ, Shaw JE, Zimmet PZ. The metabolic syndrome: prevalence in worldwide populations. Endocrinology and metabolism clinics of North America. 2004;33(2):351-75, table of contents.

24. Yang W, Reynolds K, Gu D, Chen J, He J. A comparison of two proposed definitions for metabolic syndrome in the Chinese adult population. The American journal of the medical sciences. 2007;334(3):184-9.

25. Nakanishi N, Suzuki K, Tatara K. Age-related change in relationship between white blood cell count and some features of the metabolic syndrome. Industrial health. 2004;42(3):359-68.

26. Nilsson G, Hedberg P, Jonason T, Lonnberg I, Tenerz A, Ohrvik J. White blood cell counts associate more strongly to the metabolic syndrome in 75-year-old women than in men: a population based study. Metabolic syndrome and related disorders. 2007;5(4):359-64.

27. Kirilmaz B, Asgun F, Alioglu E, Ercan E, Tengiz I, Turk U, et al. High inflammatory activity related to the number of metabolic syndrome components. Journal of clinical hypertension (Greenwich, Conn). 2010;12(2):136-44.

28. Hotamisligil GS, Shargill NS, Spiegelman BM. Adipose expression of tumor necrosis factor-alpha: direct role in obesity-linked insulin resistance. Science (New York, NY). 1993;259(5091):87-91.

29. Cinti S, Mitchell G, Barbatelli G, Murano I, Ceresi E, Faloia E, et al. Adipocyte death defines macrophage localization and function in adipose tissue of obese mice and humans. Journal of lipid research. 2005;46(11):2347-55.

30. Kim JA, Montagnani M, Koh KK, Quon MJ. Reciprocal relationships between insulin resistance and endothelial dysfunction: molecular and pathophysiological mechanisms. Circulation. 2006;113(15):1888-904.

31. Dandona P, Aljada A, Chaudhuri A, Mohanty P, Garg R. Metabolic syndrome: a comprehensive perspective based on interactions between obesity, diabetes, and inflammation. Circulation. 2005;111(11):1448-54.

32. Murano I, Barbatelli G, Parisani V, Latini C, Muzzonigro G, Castellucci M, et al. Dead adipocytes, detected as crown-like structures, are prevalent in visceral fat depots of genetically obese mice. Journal of lipid research. 2008;49(7):1562-8.

33. Borné Y, Smith JG, Nilsson PM, Melander O, Hedblad B, Engström G. Total and Differential Leukocyte Counts in Relation to Incidence of Diabetes Mellitus: A Prospective Population-Based Cohort Study. PLOS ONE. 2016;11(2):e0148963. 
34. Harrison DG, Guzik TJ, Goronzy J, Weyand C. Is hypertension an immunologic disease? Current cardiology reports. 2008;10(6):464-9.

35. Libby P. Vascular biology of atherosclerosis: overview and state of the art. The American journal of cardiology. 2003;91(3a):3a-6a.

36. Manabe S, Okura T, Watanabe S, Higaki J. Association between carotid haemodynamics and inflammation in patients with essential hypertension. Journal of human hypertension. 2005;19(10):787-91.

37. Pusuroglu H, Akgul O, Erturk M, Ozal E, Celik O, Gul M, et al. A comparative analysis of leukocyte and leukocyte subtype counts among isolated systolic hypertensive, systo-diastolic hypertensive, and non-hypertensive patients. Kardiologia polska. 2014;72(8):748-54.

38. Cnop M, Havel PJ, Utzschneider KM, Carr DB, Sinha MK, Boyko EJ, et al. Relationship of adiponectin to body fat distribution, insulin sensitivity and plasma lipoproteins: evidence for independent roles of age and sex. Diabetologia. 2003;46(4):459-69.

39. Lovren F, Pan Y, Quan A, Szmitko PE, Singh KK, Shukla PC, et al. Adiponectin primes human monocytes into alternative anti-inflammatory M2 macrophages. American journal of physiology Heart and circulatory physiology. 2010;299(3):H656-63.

40. Sica A, Mantovani A. Macrophage plasticity and polarization: in vivo veritas. The Journal of clinical investigation. 2012;122(3):787-95.

41. Sankhla M, Sharma TK, Mathur K, Rathor JS, Butolia V, Gadhok AK, et al. Relationship of oxidative stress with obesity and its role in obesity induced metabolic syndrome. Clinical laboratory. 2012;58(5-6):385-92.

42. Bremer AA, Devaraj S, Afify A, Jialal I. Adipose tissue dysregulation in patients with metabolic syndrome. The Journal of clinical endocrinology and metabolism. 2011;96(11):E1782-8.

43. Menart-Houtermans B, Rütter R, Nowotny B, Rosenbauer J, Koliaki C, Kahl S, et al. Leukocyte Profiles Differ Between Type 1 and Type 2 Diabetes and Are Associated With Metabolic Phenotypes: Results From the German Diabetes Study (GDS). Diabetes Care. 2014;37(8):2326.

44. Grossmann V, Schmitt VH, Zeller T, Panova-Noeva M, Schulz A, Laubert-Reh D, et al. Profile of the Immune and Inflammatory Response in Individuals With Prediabetes and Type 2 Diabetes. Diabetes Care. 2015;38(7):1356-64.

45. Itariu BK, Stulnig TM. Autoimmune aspects of type 2 diabetes mellitus - a mini-review. Gerontology. 2014;60(3):189-96.

46. Donath MY, Shoelson SE. Type 2 diabetes as an inflammatory disease. Nature reviews Immunology. 2011;11(2):98-107.

47. van Diepen JA, Thiem K, Stienstra R, Riksen NP, Tack CJ, Netea MG. Diabetes propels the risk for cardiovascular disease: sweet monocytes becoming aggressive? Cellular and Molecular Life Sciences. 2016;73(24):4675-84.

48. Mian MO, Paradis P, Schiffrin EL. Innate immunity in hypertension. Current hypertension reports. 2014;16(2):413. 


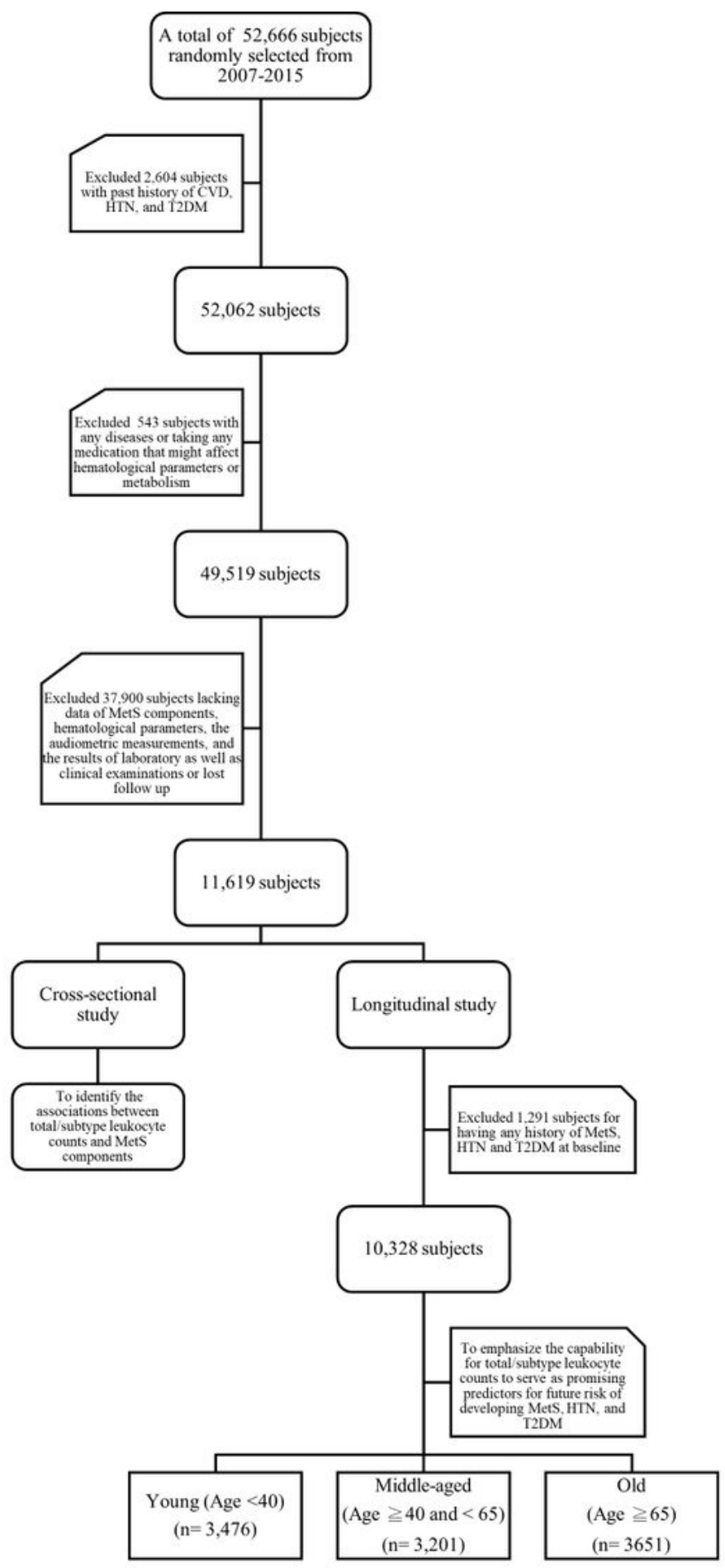

Figure 1

Description of the study design. Abbreviation: CVD, cardiovascular disease; HTN, hypertension; MetS, metabolic syndrome; T2DM, type 2 diabetes mellitus. 


\section{Supplementary Files}

This is a list of supplementary files associated with this preprint. Click to download.

- Supplementarytable.docx 